\title{
Apollonia d'Illyrie (Albanie)
}

Campagne de fouilles 2012

Jean-Luc Lamboley, Faïk Drini, François Quantin, Stéphane Verger, Altin Skenderaj et Saïmir Shpuza

\section{(2) OpenEdition}

Édition électronique

URL : http://journals.openedition.org/cefr/1040

DOI : $10.4000 /$ cefr. 1040

ISSN : 2282-5703

Éditeur

École française de Rome

\section{Référence électronique}

Jean-Luc Lamboley, Faïk Drini, François Quantin, Stéphane Verger, Altin Skenderaj et Saïmir Shpuza, « Apollonia d'Illyrie (Albanie) », Chronique des activités archéologiques de l'École française de Rome [En ligne], Balkans, mis en ligne le 14 janvier 2014, consulté le 01 mai 2019. URL : http:// journals.openedition.org/cefr/1040 ; DOI : 10.4000/cefr.1040

Ce document a été généré automatiquement le 1 mai 2019.

(c) École française de Rome 


\title{
Apollonia d'Illyrie (Albanie)
}

\author{
Campagne de fouilles 2012
}

Jean-Luc Lamboley, Faïk Drini, François Quantin, Stéphane Verger, Altin Skenderaj et Saïmir Shpuza

1 Les crédits alloués en 2012 par le MAEE et complétés par la contribution de l'EFR ont permis de financer une mission de deux semaines du 9 au 21 avril, entièrement consacrée au traitement du matériel, et une mission de quatre semaines du 6 août au $1^{\mathrm{er}}$ septembre au cours de laquelle, comme les années précédentes, les fouilles ont porté sur le portique nord de l'agora dans la ville haute, et sur le grand égout du secteur $\mathrm{G}$ dans la ville basse (fig. 1). 
Fig. 1 - Apollonia d'Illyrie. Localisation des deux zones de fouille.

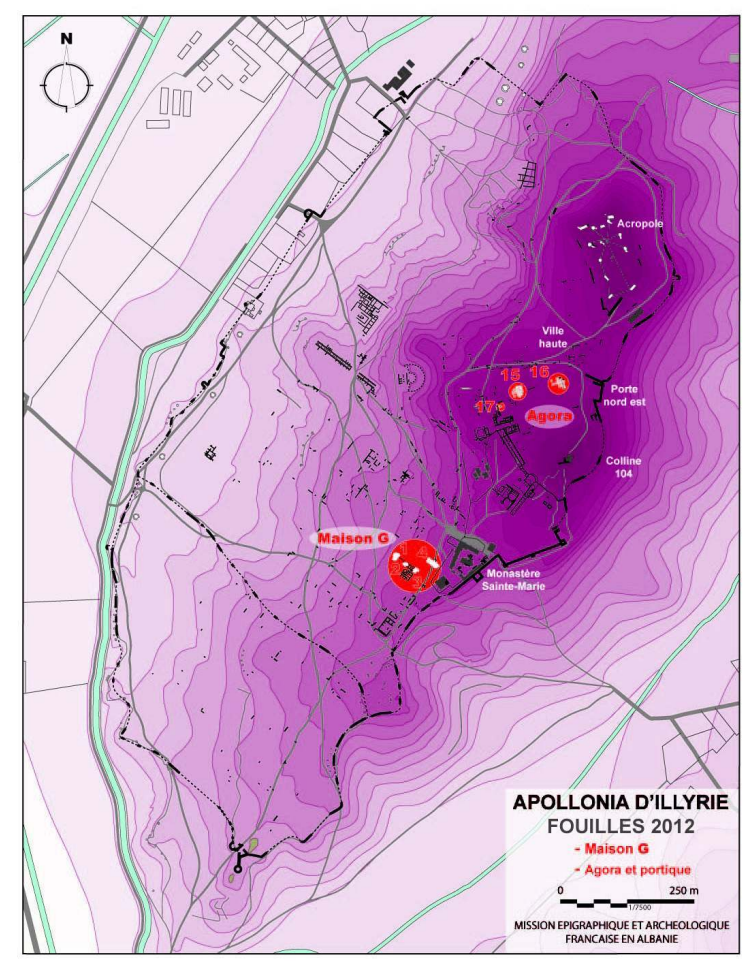

2 Parallèlement, l'Institut des Monuments de Tirana, sous la conduite de A. Islami, a poursuivi la restauration des mosaïques du péristyle de la maison à impluvium du secteur $G$ et de l'édifice voisin découvert en 2011. Comme les années précédentes, un remerciement va à l'INRAP pour la mise à disposition de l'architecte $\mathrm{Ph}$. Lenhardt, et à E. Follain archéologue de la DRAC de Rouen.

\section{LA VILLE HAUTE : FOUILLE DU GRAND PORTIQUE (S. Verger, F. Quantin, S. Shpuza, avec la participation de Th. Contamine, A. Keturakis et E. Tanka,)}

Dans le secteur 15 (fig. 2), on a fini de dégager l'édifice semi-circulaire découvert par la prospection électrique en 2004, et définitivement établi le lien stratigraphique qu'il entretient avec le mur du fond du portique. On a également mis au jour les bases de colonnes 16 et $17 \mathrm{du}$ portique, et étendu vers le nord-est la fouille des niveaux de la première moitié du $\mathrm{VI}^{\mathrm{e}}$ siècle avant $\mathrm{J}$.-C. 
Fig. 2 - Apollonia d'Illyrie. Position des secteurs 15, 16 et 17 sur le plan des prospections électriques.

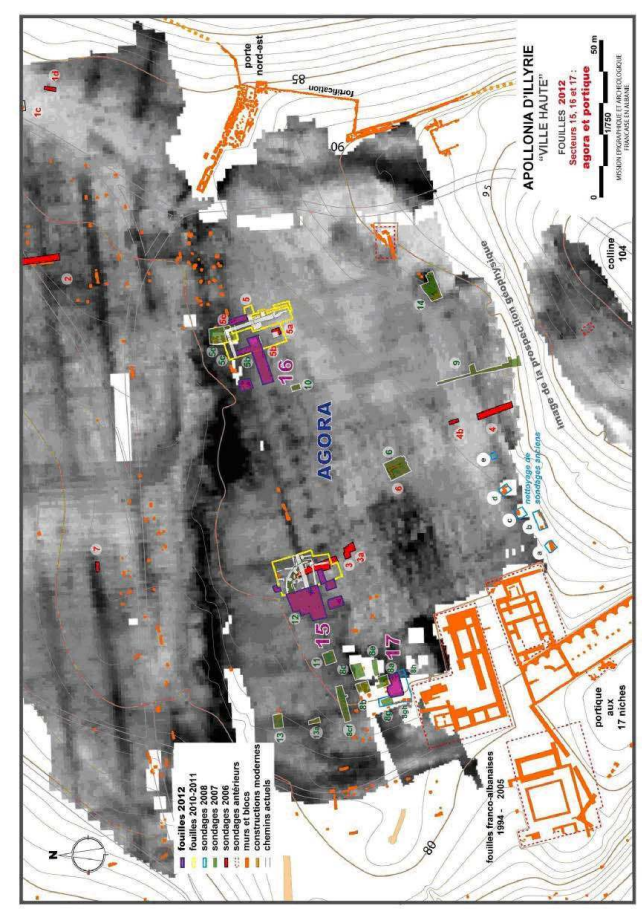

Dans le secteur 16 (fig. 2), on a fouillé dans la zone décapée à la pelle mécanique à la fin de la campagne de 2011 pour dégager les bases de colonne 3 et 4 et on a effectué des sondages le long du mur du fond du portique afin de fouiller les niveaux anciens sous les bases et de préciser l'orientation des îlots archaïques. Dans le secteur 17 (fig. 2), on a entièrement mis au jour l'angle sud-ouest du portique et on a découvert les restes d'une rue est-ouest plus ancienne ainsi qu'un remblai très riche en matériel archaïque de tout le $\mathrm{VI}^{\mathrm{e}}$ siècle avant $\mathrm{J}$.-C. Ainsi, la campagne de 2012 a apporté des réponses à diverses questions posées en début de programme. Les résultats obtenus seront présentés ici par ordre chronologique, sans tenir compte de la division en secteurs, afin de privilégier une approche synoptique.

\section{Avant le grand portique hellénistique (du début du VI siècle au II siècle avant J.-C.)}

\section{Les quartiers bleus : topographie générale et chronologie}

Les prospections électriques avaient précisé les caractéristiques de l'organisation urbaine la plus ancienne de la ville haute d'Apollonia, qui avait été confirmée par les sondages topographiques. Les fouilles du quartier de l'agora ont permis de préciser ces données sur trois points: le rythme des îlots et des rues; la coexistence de plusieurs quartiers organisés selon le même rythme mais présentant des orientations différentes; la chronologie de la mise en place et de l'abandon du plan urbain ancien. 
Sur le premier point, la fouille de 2012 a été très instructive. Dans le secteur 15 , on a pu pour la première fois dégager dans toute leur largeur un îlot et les deux rues qui le bordaient (fig. 3).

Fig. 3 - Apollonia d'Illyrie. Plan du secteur 15.

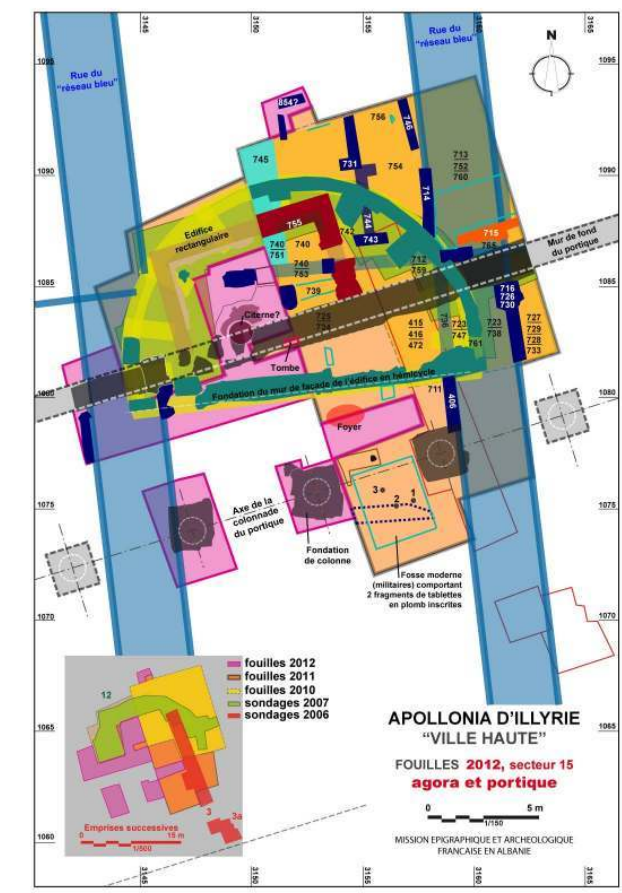

7 La largeur originelle précise de ces différents éléments n'est pas facile à établir. D’une part, les murs de limite d'îlot ont subi plusieurs réfections et leurs différents états se superposent de manière approximative. On observe généralement, de l'époque tardoarchaïque à l'époque hellénistique, un élargissement de l'îlot aux dépens des rues, c'est-àdire un empiètement de l'espace privé sur l'espace public. L'état le plus ancien des murs, lorsqu'il est conservé, est difficilement accessible sous les états postérieurs. D'autre part, dans plusieurs secteurs, les murs de limite d'îlot ont disparu et leurs blocs sont réemployés.

La compilation des données disponibles permet tout de même de proposer une hypothèse quant aux mesures utilisées pour l'implantation des îlots et des rues. Sur l'état le plus ancien documenté du mur ouest de l'îlot, une ligne tracée sur le lit d'attente d'un bloc de fondation pour la mise en place de l'assise supérieure indique que ces mesures étaient très précises. La largeur de la rue ouest atteint environ 3,25 mètres. Celle de l'îlot, murs de limite compris, est de 13 mètres. Si l'on suppose que le pied utilisé mesurait 0,325 mètre, on peut ainsi émettre l'hypothèse selon laquelle le plan originel consistait en une alternance de stenopoi de 10 pieds et d'îlots de 40 pieds. 50 pieds séparent donc les axes de deux sténopoi voisins. Cette mesure est anormalement réduite par rapport à ce que l'on connait de l'urbanisme grec archaïque. Ainsi par exemple, dans le contexte également corinthien des colonies de Syracuse en Sicile orientale, comme Kasmenai, les îlots ont une largeur double de celle que l'on observe à Apollonia. 
9 L'organisation interne des îlots est plus difficile à restituer, compte tenu de la surface relativement réduite qui a été fouillée. On dispose de quelques indices fragiles d'une division axiale, mais il peut s'agir aussi des traces d'une division interne d'une maison. On manque encore d'indications pour évaluer la largeur des lots dans l'îlot. En 2012, une petite extension de la fouille vers le nord a permis de découvrir un mur est-ouest, c'est-àdire transversal par rapport à l'axe de lîlot qui ressemble beaucoup à un mur est-ouest trouvé en 2010 plus au sud, dont l'état classique est lié au mur de limite est de l'îlot. Or, les axes de ces deux murs sont distants de 6,5 mètres, soit 20 pieds du système indiqué précédemment. On pourrait donc proposer, à titre d'hypothèse de travail, une division des îlots en lots de 40 sur 20 pieds.

Comme l'avait montré la prospection électrique, la zone de l'agora hellénistique se trouve à proximité des limites entre trois quartiers obéissant au même rythme d'îlots et de stenopoi, mais orientés différemment : le réseau est/ouest « bleu » au nord, le réseau nordouest/sud-est « de la fortification » au nord-est et le réseau nord/sud «bleu transversal » au sud. La limite entre les deux premiers, au niveau de la grande rue qui va de la porte nord-est à la rampe de l'acropole, est claire depuis 2006. La limite entre les réseaux «bleu » et «bleu transversal » correspond à un talus qui est bien visible sur le terrain et sur la carte de la prospection électrique. Celui-ci est bien marqué à l'ouest, mais il s'atténue vers l'est, entre l'angle du portique hellénistique et la porte nord-est.

Les fouilles de 2010-2012 apportent quelques indications quant à l'extension vers l'est et vers le sud du réseau «bleu transversal ». En effet, en 2012, la fouille du secteur 16 a mis en évidence un stenopos orienté nord-sud entre les bases de colonne 2 et 3 du portique hellénistique, qui ont recouvert les deux murs de limite. Le mur ouest est bien visible sous la fondation de la base 3 . Une couche de petits galets mise en évidence à l'est, jusqu'à la base 2, indique la présence d'un niveau de rue. Près de l'angle sud-ouest de la base 2, on pourrait enfin avoir la trace de la tranchée de spoliation du mur est de la rue, qui aurait dans ce cas une largeur d'environ 3 mètres. Le mur ouest de cette rue se trouve à une distance d'environ 64 mètres du mur est de la rue est du secteur 15. Cela représente la largeur cumulée de quatre îlots et trois stenopoi. Le stenopos mis au jour dans le secteur 16 appartient donc bien au réseau bleu transversal.

La combinaison des informations livrées sur le réseau "bleu transversal» par les prospections de 2004-2005, les sondages de 2006-2008 et les fouilles de 2010-2012 permet de restituer de façon certaine 7 îlots et les 8 stenopoi qui les bordent, qui s'étendent sur presque toute la longueur du portique hellénistique, qui les recouvre. Plus à l'ouest, la prospection électrique indique qu'il existe encore au moins un îlot. À l'est, la situation est plus compliquée et demanderait un complément de fouille.

13 Vers le sud, les structures antérieures au portique hellénistique ont été détruites par les travaux d'aménagement de l'agora, qui ont nécessité d'entailler le flanc nord de la colline 104. C'est ce qu'avaient montré les sondages de 2006-2007 dans cette zone. En 2012, la fouille du secteur 17 a mis en évidence deux murs est-ouest antérieurs au portique hellénistique, qui sont distants entre eux d'environ 3 mètres. Le long du mur sud était conservé un lambeau de strate de galets semblable à celui qui marque les niveaux de rue. On peut ainsi faire l'hypothèse qu'il $\mathrm{y}$ avait une rue transversale au système «bleu transversal » là où plus au nord, d'après le sondage $8 \mathrm{f}$, se trouvait l'îlot d'habitations. On aurait affaire à une rue transversale qui reliait les stenopoi entre eux. Il pourrait être intéressant à l'avenir de tenter de la retrouver plus à l'est pour comprendre si elle constitue un simple axe transversal ou bien sépare deux quartiers différents. Quoiqu'il en 
soit, la distance entre le talus au nord et cette rue est environ de 50 mètres, ce qui est maintenant l'extension minimale connue du quartier « bleu transversal» du nord au sud. Il faudrait reprendre en détail à la lumière de ces résultats les données provenant de la fouille de 1994-2006, qui a aussi livré des vestiges d'orientation «bleue transversale ».

Dans le secteur 15, la fouille de 2011 a montré que le réseau bleu transversal était en place au moins depuis la période tardo-archaïque. Le premier état conservé du mur de limite est de l'îlot est lié stratigraphiquement au remblai de cette époque. Ce résultat a été confirmé en 2012 dans le secteur 16. La fondation du mur ouest du stenopos était également associée à un remblai qui contient une abondante série céramique $\mathrm{du} \mathrm{VI}^{\mathrm{e}}$ et du début $\mathrm{du} \mathrm{V}^{\mathrm{e}}$ siècle avant J.-C. Plusieurs indices semblent montrer que l'installation du réseau «bleu transversal », comme celle du réseau «bleu ", remonte à une époque plus ancienne, peut-être contemporaine des débuts de la colonie. En 2011, on avait fouillé un trou de poteau dont l'orientation était parallèle au mur de limite de l'îlot et dont le remplissage contenait une série céramique très homogène des environs du début du $\mathrm{VI}^{\mathrm{e}}$ siècle avant J.-C. Cette année, plus au sud dans l'îlot, on a pu mettre en évidence deux tranchées de spoliation de murs est-ouest remplies par du matériel archaïque (dont des amphores de transport), ce qui suggère que leur construction remontait elle aussi à la première moitié du $\mathrm{VI}^{\mathrm{e}}$ siècle. C'est en effet la date livrée par la céramique associée au premier sol de maison, dont une zone probable de foyer, autour de ces murs. Malheureusement, l'état très dégradé des structures construites dans les niveaux les plus anciens ne permet pas d'être plus précis sur l'organisation de l'îlot pendant cette phase ancienne.

À ces deux phases archaïques - un niveau de sol et un remblai - succède, dans l'îlot du secteur 15, un remblai classique des environs de 400 avant J.-C., puis, dans tous les secteurs, un remblai hellénistique dont le matériel est majoritairement de la fin du IV et de la première moitié du $\mathrm{III}^{\mathrm{e}}$ siècle avant $\mathrm{J} . \mathrm{-}$ - . Une étude exhaustive de la céramique très abondante de cette strate est indispensable pour établir la date de cette phase qui pourrait descendre jusqu'au début du II siècle avant J.-C. Dans le secteur 15, c'est à cette phase que sont associés les murs à élévation de fragments de tuiles, qui forment des cloisons mais aussi un état tardif des murs de limite d'îlot. Certains de ces murs sont taillés par les fondations de l'édifice semi-circulaire et le remblai hellénistique et couvert par la canalisation qui borde le grand portique. L'îlot a ainsi subi différentes destructions lors des phases récentes de l'époque hellénistique.

En 2012, dans le secteur 15, la fouille de la tranchée de spoliation du mur du fond du portique, qui contenait les blocs déplacés de la première assise de fondation et une grande quantité de fragments architecturaux, a conduit à la découverte de l'embouchure circulaire d'un puits ou d'une citerne (fig. 4). 
Fig. 4 - Apollonia d'Illyrie. Orifice du puits dans le secteur 15.

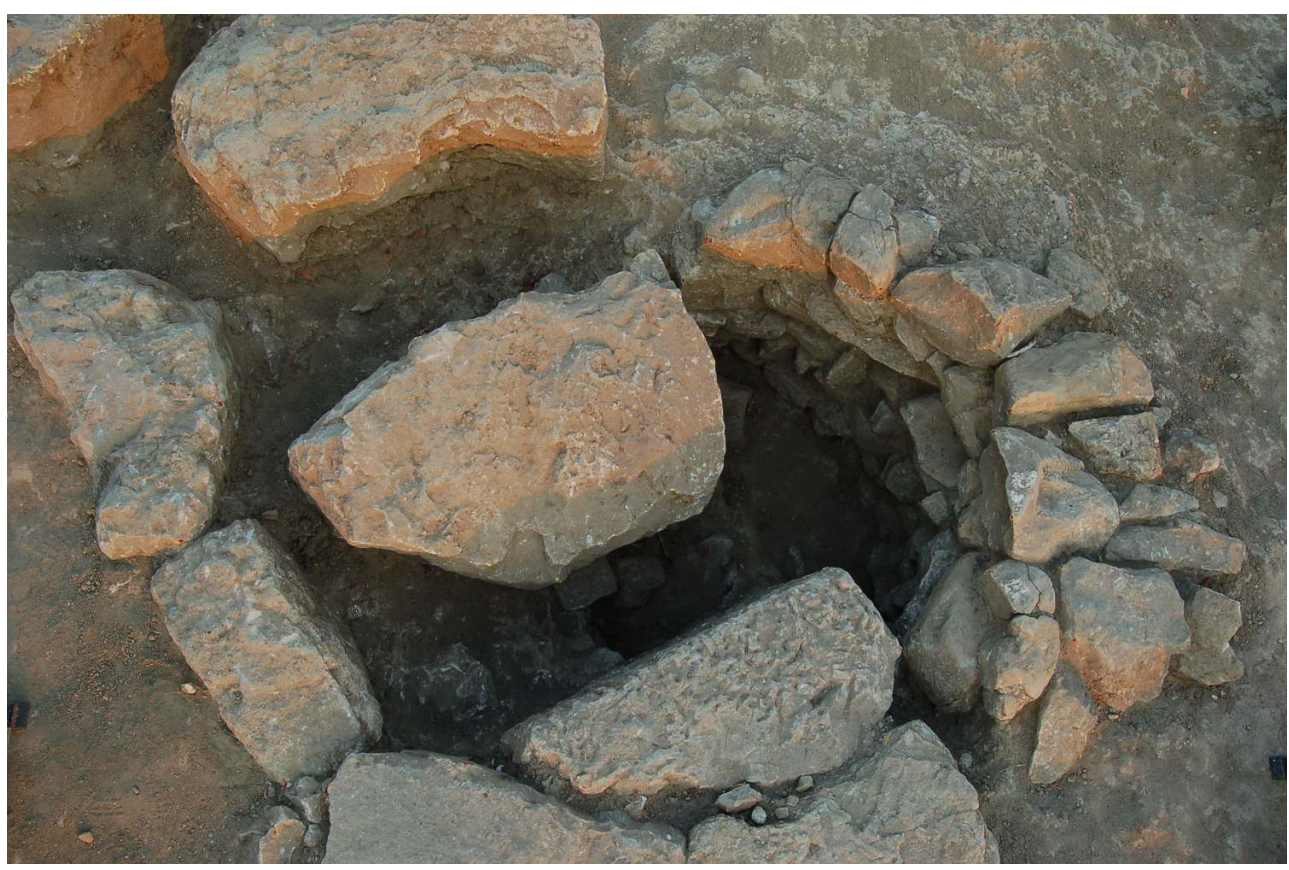

Le conduit est construit en moellons bruts. Le diamètre interne atteint 1,4 m. Le sommet du remplissage, qui a été fouillé, contient quelques fragments céramiques, parmi lesquels des tessons de vases à vernis noir comparables à ceux du remblai hellénistique. La clôture complète de la structure pourrait donc être datée du III ${ }^{e}$ siècle avant J.-C. Elle est antérieure à la construction du portique, puisque la margelle est taillée par la tranchée de fondation du mur du fond et couverte par la première assise de fondation. Les moellons de l'assise supérieure de la margelle reposent sur un sol recouvert par le remblai tardoarchaïque. Seule la fouille complète de cette structure permettra d'en déterminer la fonction (puits ou citerne), la datation et la fonction (privée ou publique). Le meilleur parallèle provient du secteur 16 où, en 2011, avait été mise au jour une margelle circulaire plus petite mais dont le remplissage semble contemporain. Comme celle du secteur15, cette citerne (ou ce puits) est associée à un mur du réseau « bleu transversal » et doit se trouver dans un îlot d'habitation. La fouille en serait plus délicate, en raison de son diamètre plus restreint.

Parmi le matériel récupéré cette année dans le secteur on signalera deux graffites :

- un fond de skyphos à vernis noir (fig. 5) de la première moitié du $\mathrm{V}^{\mathrm{e}}$ siècle avant $\mathrm{J}$.-C., avec une inscription, où il faut lire vraisemblablement MOM, c'est-à-dire l'abréviation du nom Mommios (CIGIME I-1, $\mathrm{n}^{\circ}$ 315). Il s'agit de l'indication du propriétaire de cette coupe. 
Fig. 5 - Apollonia d'Illyrie. Graffite sur fond de skyphos.

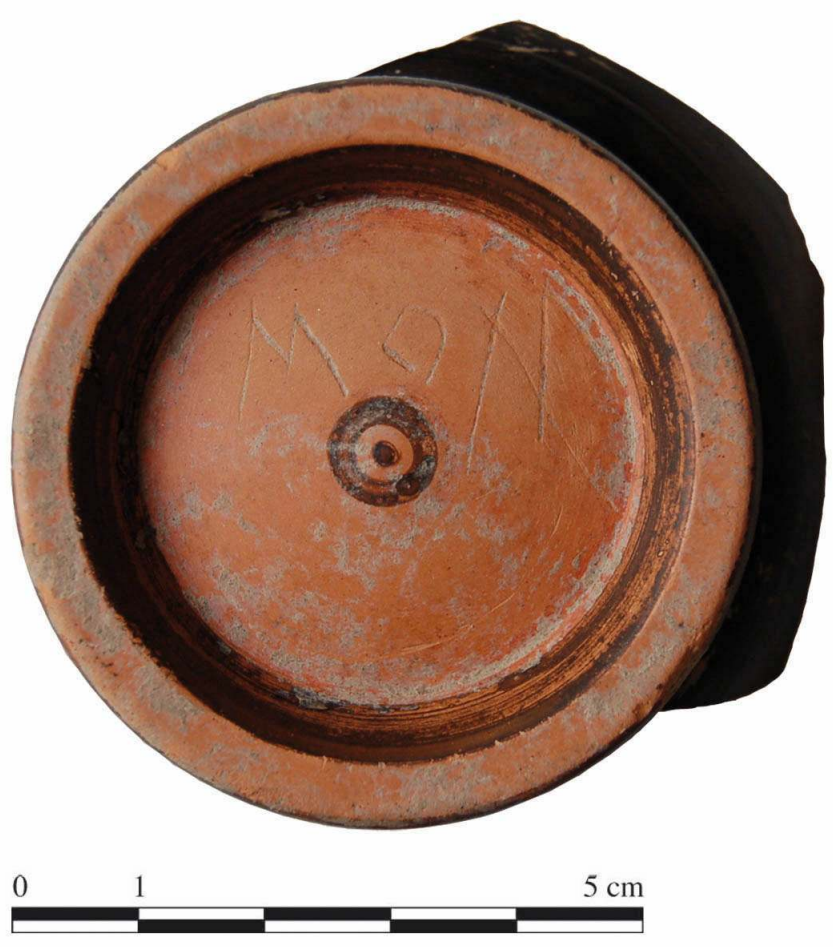

- un fragment d'assiette à vernis noir (fig. 6) de la première moitié du III siècle avant J.-C. avec une inscription incisée. On lit un verbe, sans doute une forme de ó $\varphi \varepsilon\{1 \lambda \omega$, « avoir une dette », suivi d'un nombre, $210(\mathrm{HH} \Delta)$ ou 310 (HHH $\Delta)$. Il s'agit donc manifestement d'une petite note destinée à conserver le souvenir d'une dette. Cette courte inscription est à sa place sur une agora. 
Fig. 6 - Apollonia d'Illyrie. Graffite sur assiette à vernis noir.

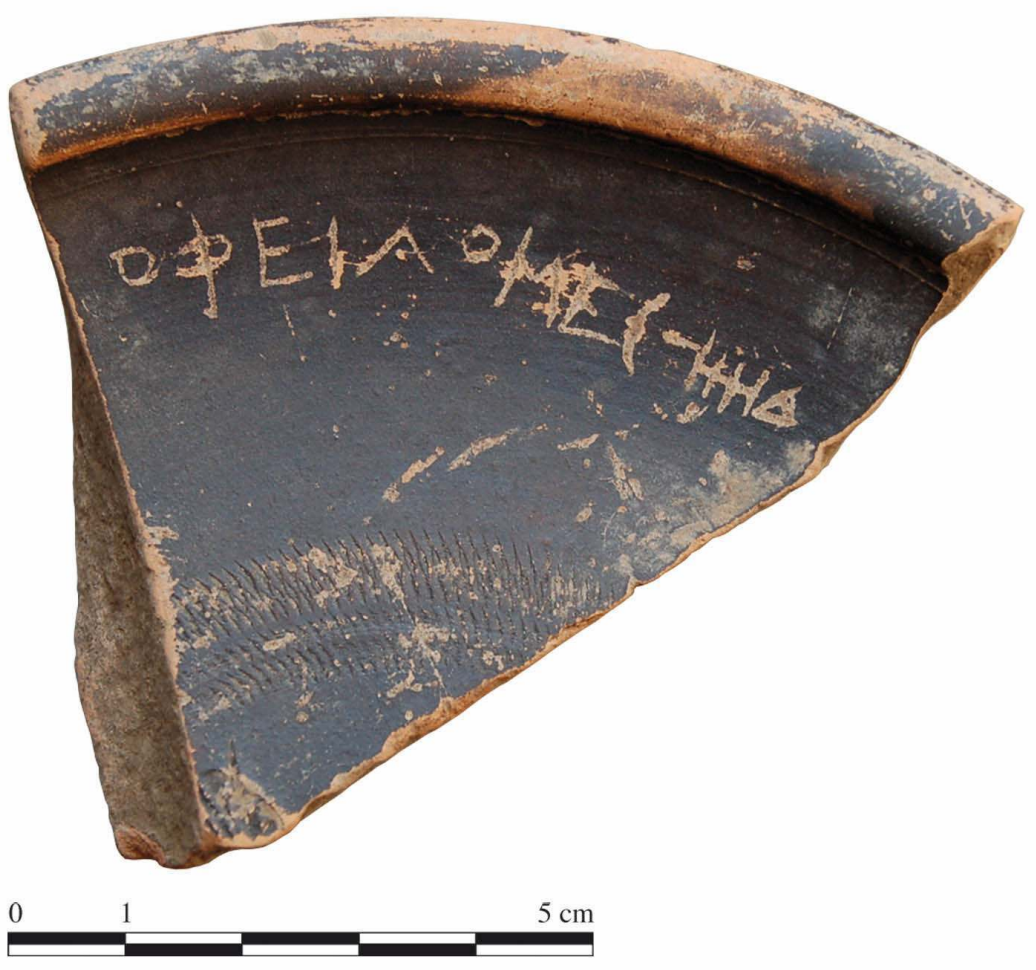

Le bâtiment semi-circulaire (fig. $3,7,8$ )

19 La fouille de 2012 dans le secteur 15 a permis de mettre au jour l'ensemble du bâtiment semi-circulaire qui recoupe l'îlot en détruisant partiellement les murs qui le bordent et les parois internes. 
Fig. 7 - Apollonia d'Illyrie. L'édifice circulaire vu de l'est.

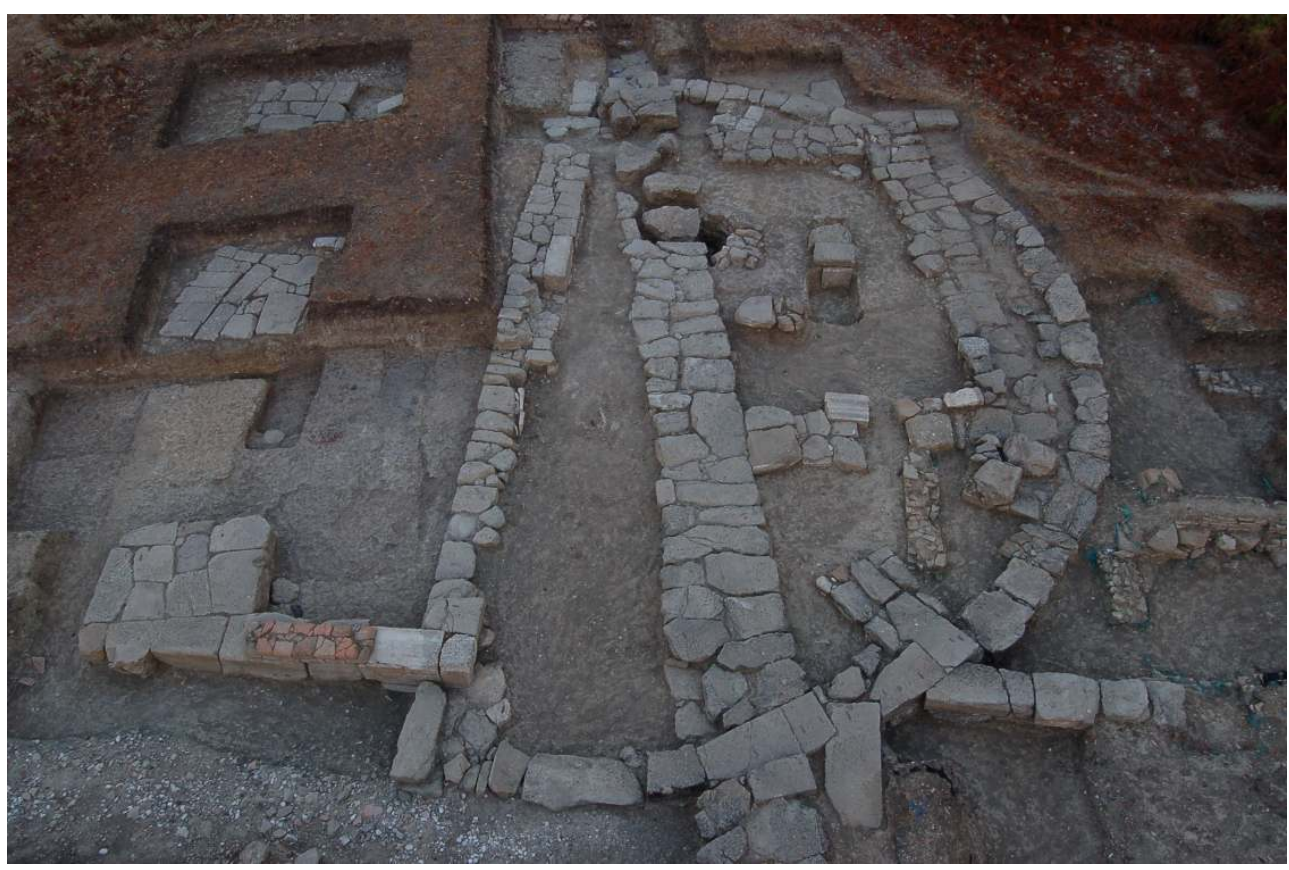

Fig. 8 - Apollonia d'Illyrie. L'édifice semi-circulaire vu du sud (secteur 15).

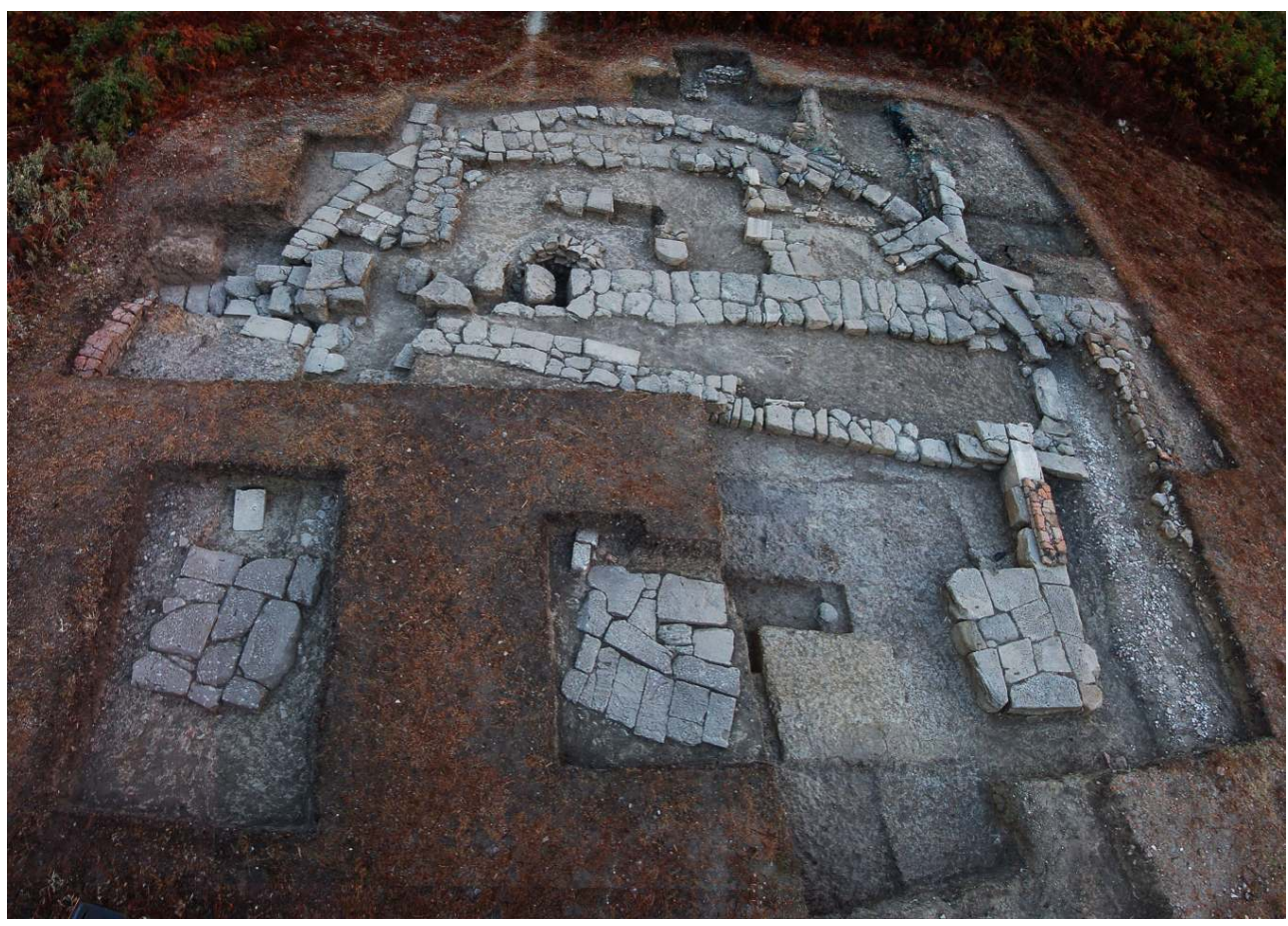

20 L'édifice s'insère toutefois dans le plan ancien puisque qu'il conserve l'orientation « bleue transversale » et est disposé symétriquement par rapport à l'axe longitudinal de l'îlot. Les extrémités est et ouest mordent sur les rues adjacentes sans toutefois les fermer complètement. Le mur de façade est maintenant entièrement dégagé, ainsi que l'angle sud-ouest. On peut supposer au sud l'existence d'un avant-corps rectangulaire qui a presque entièrement disparu en raison des travaux effectués au moment de la 
construction du portique. Le mur oriental, qui est connu depuis 2006, a maintenant son symétrique à l'ouest, même si ce n'est que sous la forme d'une tranchée de spoliation. En revanche, on n'a pu trouver aucune trace certaine du mur sud. Il existe bien là aussi une tranchée de spoliation d'un mur ancien, mais qui semble dater de l'époque archaïque, comme on l'a indiqué précédemment.

\section{Conclusions}

21 La chronologie relative des bâtiments d'époque hellénistique a pu être confirmée de manière certaine. La séquence est la suivante :

- Édifice quadrangulaire très mal connu car documenté uniquement par des tranchées de spoliation de murs, dont un mur est-ouest divisant l'îlot dont il reste encore quelques blocs en place, qui sont des remplois d'un édifice d'époque archaïque ou classique. Le puits ou la citerne pourrait avoir été en fonction à l'époque de ce premier bâtiment et avoir été rempli au moment de la construction de l'édifice semi-circulaire.

- Édifice semi-circulaire avec avant-corps au sud qui détruit l'édifice rectangulaire précédent et une partie des habitations de l'îlot, plus au nord. La façade de la partie semi-circulaire a une longueur d'environ 16 mètre et l'édifice mesure 9,75 mètre du nord au sud sans l'avant corps, dont la largeur est celle de lîlot et la profondeur est inconnue. Ainsi, la partie semicirculaire mesure 50 pieds sur 30 et l'avant-corps a une largeur de 40 pieds. On connait maintenant les neuf murs radiaux qui partent du mur semi-circulaire vers l'intérieur du bâtiment.

- Portique qui détruit l'édifice semi-circulaire et les restes de l'îlot et des rues. Le lien stratigraphique entre le mur du fond du portique et l'édifice semi-circulaire est maintenant bien clair, puisque dans l'angle sud-ouest de celui-ci, l'assise inférieure de sa fondation passe sous la fondation du mur du portique. La zone où les deux bâtiments se superposent correspond d'ailleurs à un changement d'altitude du niveau inférieur de la fondation du portique, qui est plus profond à l'ouest qu'à l'est.

- Bâtiment rectangulaire adossé à la face nord du mur du fond du portique. Il est postérieur à l'édifice semi-circulaire, dont il taille la fondation en plusieurs points : cette année a été mis au jour un mur radial partiellement démonté au moment de la mise en place de l'angle nordouest. L'assise de fondation conservée est adossée à celle du mur du portique. La construction du bâtiment est donc contemporaine à celle du portique ou bien postérieure. On a pu observer cette année que le corps de bâtiment rectangulaire était disposé symétriquement par rapport aux colonnes 15-17, ce qui laisse supposer qu'il faisait partie du portique.

Pour proposer une chronologie absolue de ces différentes phases, on devra attendre l'étude complète des séries céramiques des remblais hellénistiques et des ensembles de fragments architecturaux provenant des fosses et tranchées de fondation du mur et des bases du portique, qui semblent pouvoir appartenir à plusieurs bâtiments différents et successifs. On ne se prononcera pas non plus sur la fonction du bâtiment semi-circulaire, même si l'hypothèse d'un petit bâtiment de réunions est peut-être la plus convaincante. La fouille de 2012 dans le secteur 15 a livré de nouvelles indications sur la destruction du portique. Dans la partie ouest de l'îlot se trouve une zone entièrement bouleversée où la première assise du mur avait été elle-même arrachée. Les interstices entre les blocs déplacés étaient remplis de fragments plus ou moins importants de décors architecturaux en calcaire très fragiles, parmi lesquels plusieurs pièces de chapiteaux. D'autre part, une deuxième tombe d'enfant d'époque romaine se trouvait à proximité de celle qui avait été 
fouillée l'année dernière. L'absence de mobilier associé empêche de la dater. La tombe de 2011 reste donc le meilleur élément chronologique pour la destruction du portique, qui est à situer dans la seconde moitié du II ${ }^{\mathrm{e}}$ ou la première moitié du III ${ }^{\mathrm{e}}$ siècle après J.-C.

\section{Nouvelles données sur l'architecture du grand portique}

Il est acquis que le portique comptait 23 colonnes de façade, 21 dans l'axe longitudinal est-ouest, deux en face des colonnes des extrémités est et ouest, qui forment façade des deux courtes avancées latérales (fig. 9).

Fig. 9 - Apollonia d'Illyrie. Le portique nord de l'agora.

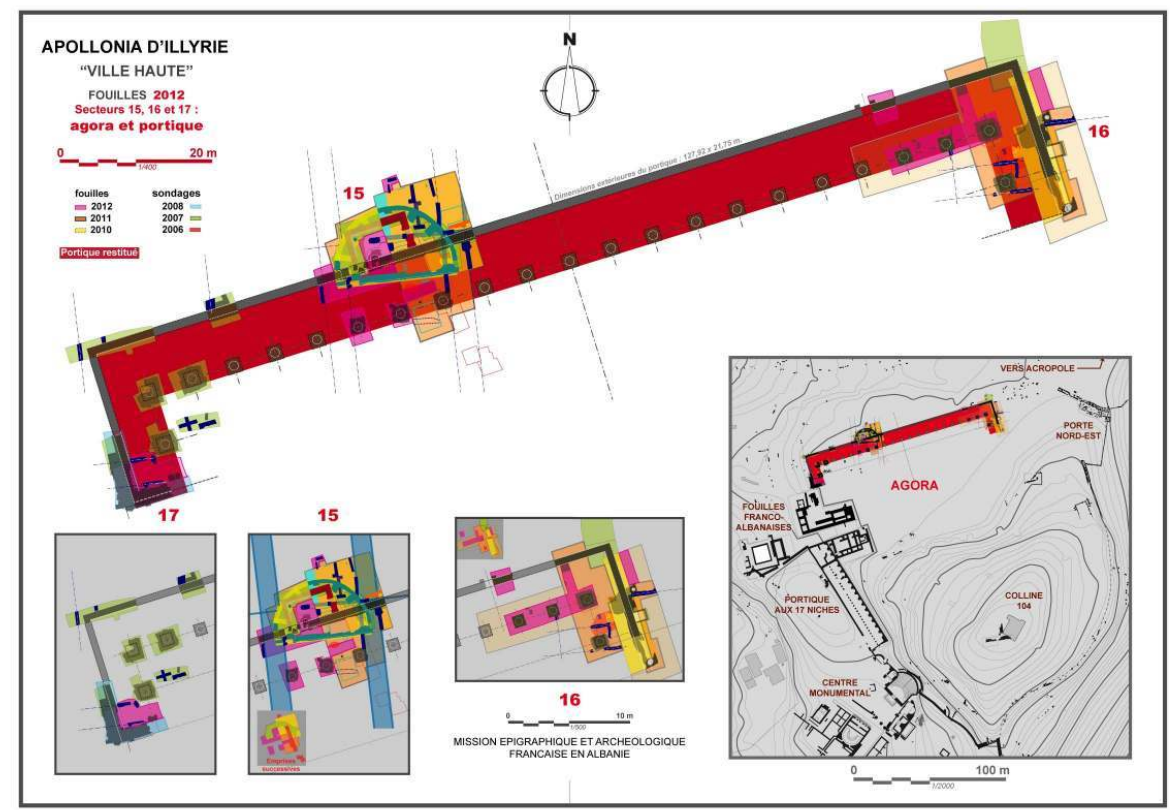

Ses dimensions sont remarquables, environ 128 x 22 m. L'un des objectifs de l'année 2012 était de vérifier la régularité de l'implantation et de la construction des fondations des colonnes en façade du portique dans les secteurs 15 et 16, et de mieux connaître le mur de fond et l'avancée occidentale (secteurs 16 et 17). Les fondations existent bien là où les indiquaient les prospections géophysiques, mais il s'avère que leur construction diffère dans les secteurs 15 et 16. Les bases du secteur 15 sont effet construites de manière moins régulière, et parfois moins soignée. Comme les années précédentes, la récupération de nombreux fragments d'architecture, principalement dans les secteurs 15 et 16, a permis de faire progresser la restitution de l'élévation du grand portique.

Les décapages menés l'an dernier grâce à une pelle mécanique dans le secteur 16 ont permis de mettre au jour dans l'extension occidentale deux bases supplémentaires et de connaître leurs assises de fondation. Pendant la fouille on a pu observer que les blocs d'architecture étaient concentrés le long de l'axe de la colonnade, et que les fragments étaient moins nombreux près du mur de fond du portique. Cette concentration, produite par le débitage systématique des blocs décorés en petits moellons aisément transportables, correspond au remplissage des tranchées de récupération des 
substructions des colonnes du portique. Aucun élément en place appartenant à la mosaïque de galets découverte en 2011 n'a été retrouvé cette année.

\section{Les fondations des bases (fig. 10, 11, 12)}

26 Les fondations des bases exhumées par la fouille et/ou signalées par la prospection géophysique sont numérotées d'est en ouest de 1 à 23 . Les bases 1 et 2 avaient été laissées à l'air libre l'an dernier. Les fondations des bases 1 à 4 sont construites de la même façon, avec quatre blocs jointifs disposés parallèlement (fig. 10).

Fig. 10 - Apollonia d'Illyrie. L'extrémité est du portique (secteur 16).

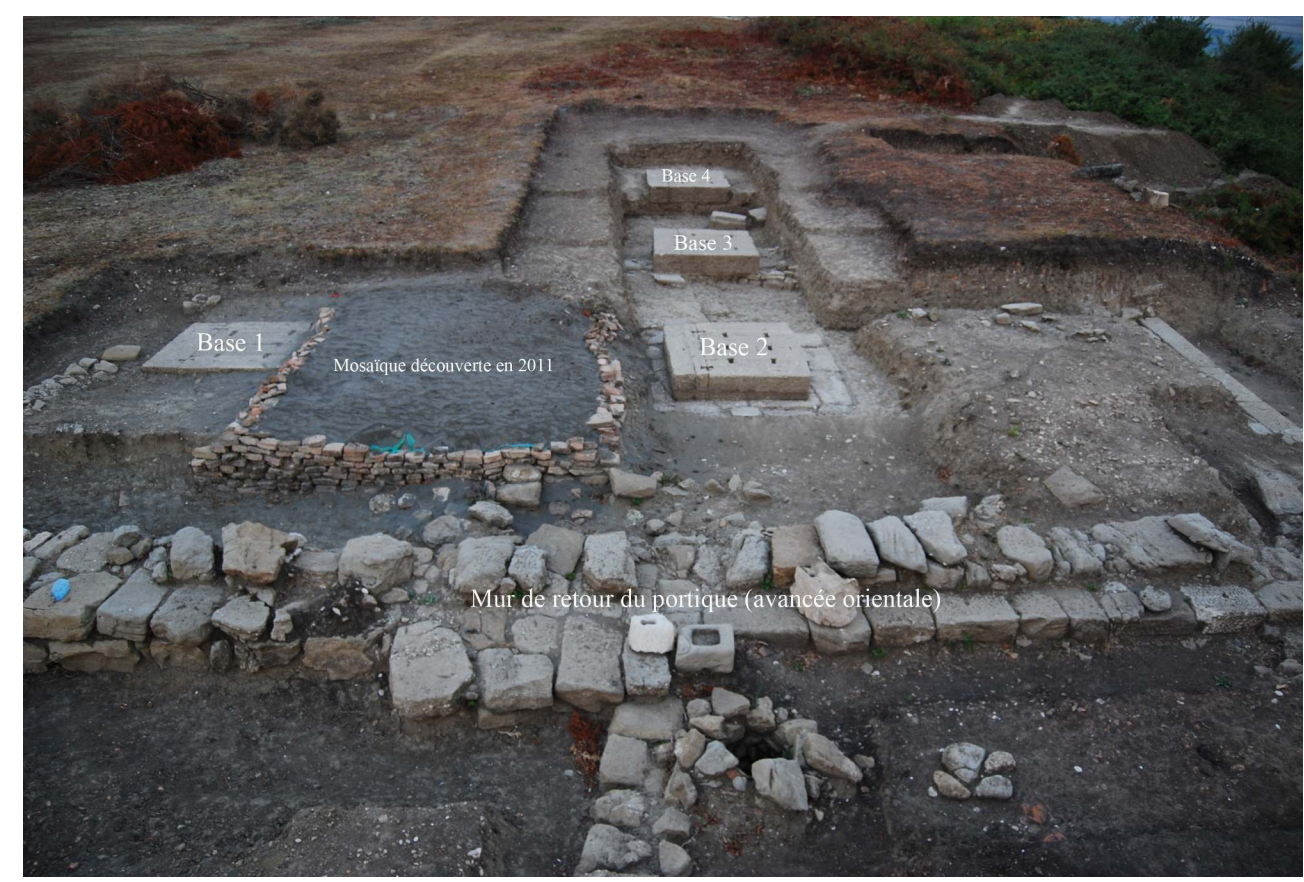

Les blocs sont assemblés grâce à des doubles queues d'aronde concaves. Des lignes de mise en place sont parfois conservées, comme des encoches de levier (souvent dites à tort "trous de pince ») destinées à permettre l'installation des blocs de l'assise supérieure disparue. La répartition de ces encoches fournit avec une grande précision le plan, la composition et la disposition des blocs de l'assise supérieure. Le lit d'attente des fondations $n^{\circ} 1$ et 2 est particulièrement riche en informations de cette nature. 
Fig. 11 - Apollonia d'Illyrie. Plan de l'extrémité orientale du portique (secteur 16).

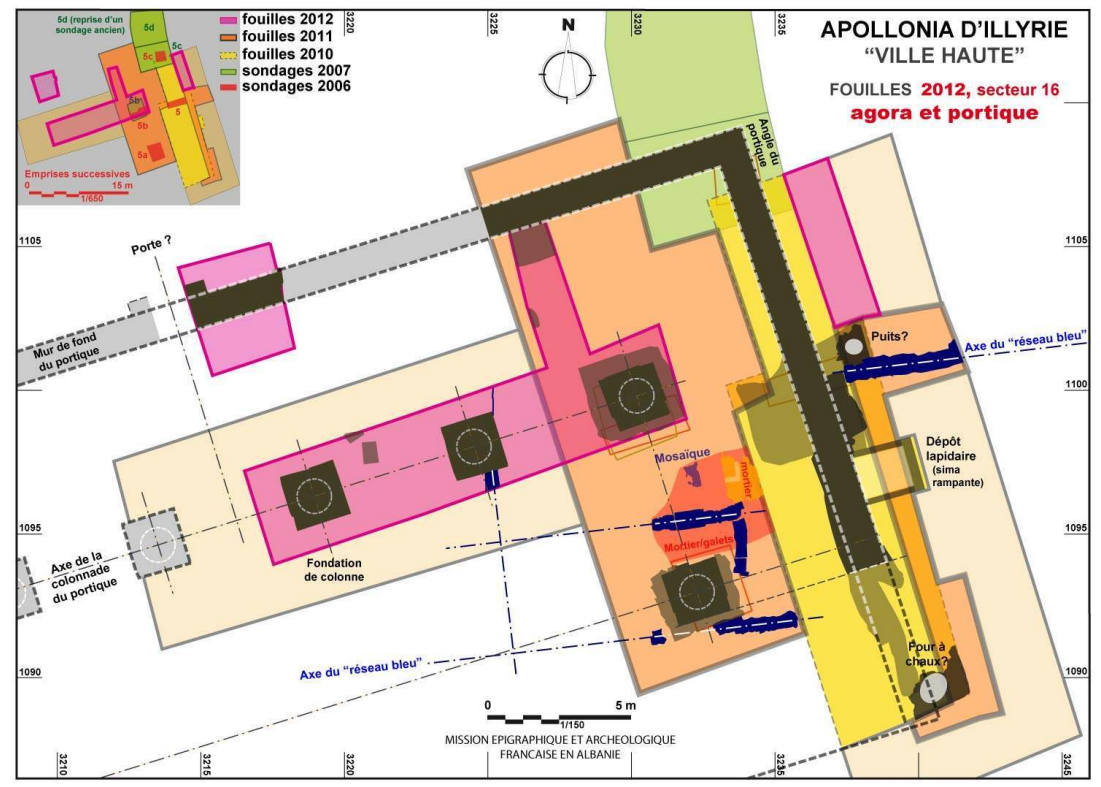

Ces bases ne paraissent pas composées de blocs de remploi, car tous les éléments d'assemblage comme les marques de mise en place sont cohérentes entre elles et s'expliquent très bien par la fonction des blocs dans la construction du portique (fig. 12).

Fig. 12 - Apollonia d'Illyrie. Les bases des colonnes du portique $(1,2,3,4,15,16)$.
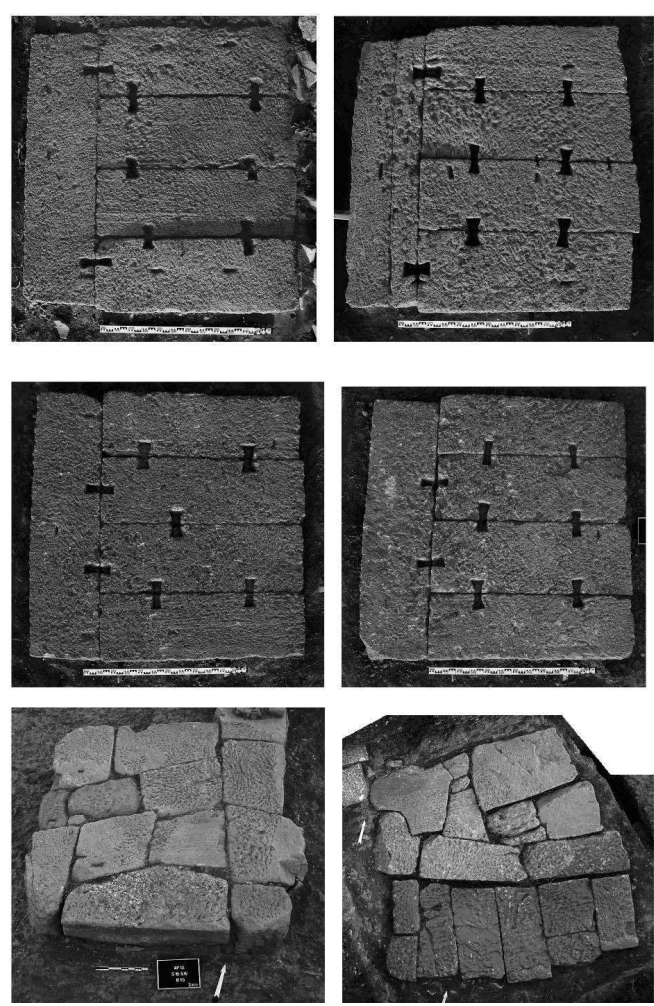

modo au même plan. La base $\mathrm{n}^{\circ} 16$ paraît être la plus effondrée, et on peut rapprocher les bases 2 et 17 autour de $27 \mathrm{~cm}$. Ces faibles variations de quelques centimètres d'altitude ne peuvent pas correspondre à une hauteur d'assise et démontrent que les fondations de bases sont toutes conservées à la même assise, malgré les différences morphologiques observées entre les bases orientales et occidentales. La base 1 accuse une différence de cote d'une vingtaine de centimètres au-dessus des bases 3, 4 et 15 . Cette différence est pour l'heure inexpliquée. La destruction de l'édifice fut particulièrement méthodique et organisée.

\section{Le mur de fond et les avancées latérales orientale et occidentale}

Dans le secteur 17 ouvert cette année, les nouvelles données concernent surtout les aménagements antérieurs à la construction du portique. Néanmoins, on connaît mieux maintenant les fondations de la façade sud de l'avancée occidentale du portique. Grâce à la découverte de blocs dans la partie est du sondage, on sait en particulier que le tracé du retour d'angle des fondations au sud-est est très vraisemblablement dans l'axe de la colonnade (fig. 13). 
Fig. 13 - Apollonia d'Illyrie. L'extrémité occidentale du portique (secteur 17).

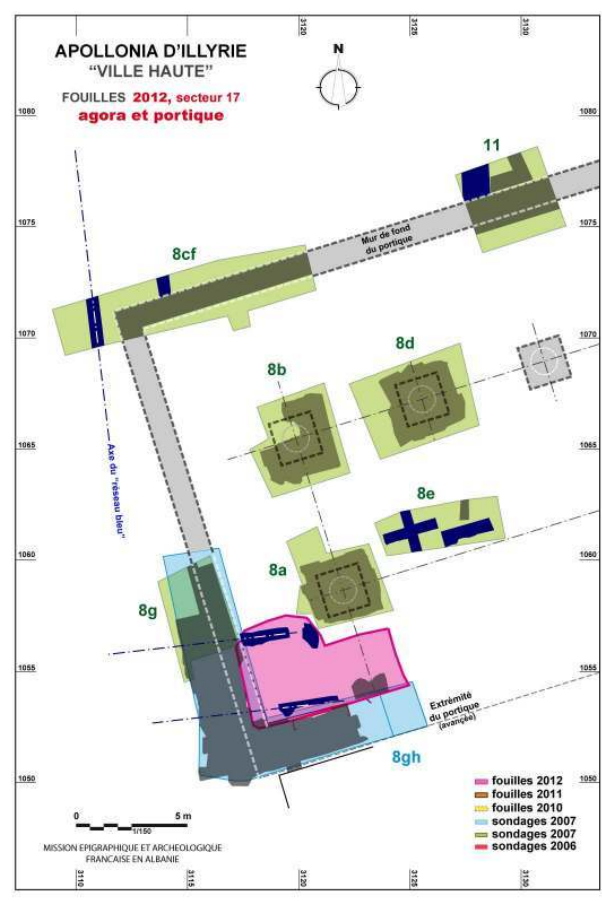

Or ce sont des informations qui sont inaccessibles à l'extrémité ouest du portique en raison des destructions subies par le monument. Le plan de l'avancée occidentale du portique est donc maintenant bien établi. Si l'on projette un mur de retour de même longueur que celui qui est attesté à l'est, il faut admettre que les blocs irréguliers dans le prolongement au sud du mur de retour correspondent aux modestes vestiges du portique dans ce secteur.

Au nord-ouest du secteur 16 il a paru opportun d'ouvrir un nouveau sondage, en profitant de l'excavation d'une ancienne tranchée nord-sud pratiquée par les militaires, au niveau grosso modo de la base 4, afin de retrouver le mur de fond du portique. Dans ce secteur, les informations nouvelles sont nombreuses.

L'extension nord, à l'arrière de la base 4 (fig. 11, 14), a permis de découvrir un nouveau tronçon de mur de fond du portique. Il est construit grâce à un appareil rectangulaire irrégulier, avec usage d'un bloc en boutisse, comme l'autre tronçon du même mur qui apparaît conservé à la même altitude dans le secteur 16 et qui forme l'angle nord-est du monument. 
Fig. 14 - Apollonia d'lllyrie. Contrefort à l'arrière du mur nord du portique (secteur 16).

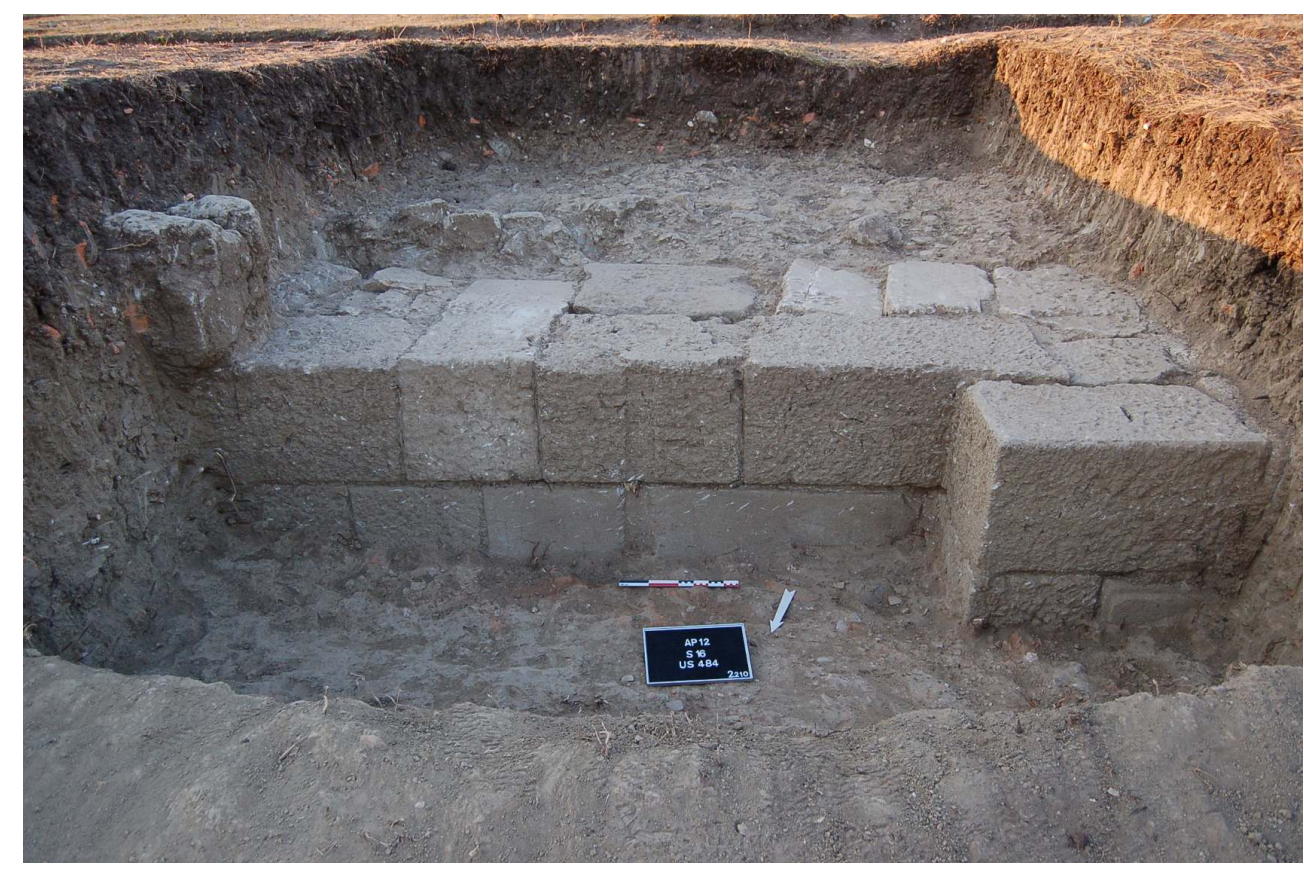

Un aspect de la construction diffère néanmoins nettement: on n'observe pas ici une longueur importante des blocs de panneresse. D'autre part, à l'arrière du mur de fond du portique, qui a incontestablement une fonction d'analemma - en raison d'une importante différence de cote entre le sol antique au nord et au sud, c'est-à-dire entre le sol des quartiers nord et le niveau de circulation à l'intérieur du bâtiment -, un blocage de petits moellons peu dégrossis et de fragments de blocs à faces travaillées qui sont assurément des remplois forme le remblai construit pour surélever la piste unique du portique. Ce remblai était moins bien conservé ailleurs dans le secteur 16, et paraît être une particularité de la structure constructive du monument dans sa partie orientale. À l'arrière de la file de parement apparait une seconde file de blocs, sûrement composée de remplois.

Au nord-ouest de l'extension nord du secteur 16 (fig. 14), est conservée sur deux assises une avancée vers le nord appareillée de la même manière que le mur. Cette avancée est vraisemblablement prévue dès la construction puisqu'elle s'encastre dans le bloc immédiatement au sud. Ce massif est encore pris dans la berme de limite de fouille à l'est. Il pourrait correspondre à un contrefort, ou bien à une particularité du mur de fond du portique, qui pourrait ici être percé d'une porte permettant de rejoindre par un emmarchement les quartiers au nord. À l'est de cette avancée, une ciselure verticale en retrait observée sur le parement nord du mur de fond du portique dans l'extension septentrionale du secteur 16 se prolonge à l'assise inférieure jusqu'au sol d'arrêt de la fouille 2012 (à l'altitude -45). Il est vraisemblable qu'elle soit présente sur l'ensemble de la hauteur de l'assise, voire qu'elle continue plus bas encore, jusqu'au bloc de la première assise aérienne du mur. Il s'agit d'une "plumée », c'est-à-dire un type de ciselure de quelques centimètres de largeur qui sert au ravalement des murs en marquant le plan vertical du parement définitif à exécuter. Dans le rempart est, où le socle en grand appareil fait de blocs de même pierre et de même forme que ceux du mur de fond du portique reçoit l'élévation de briques cuites du mur d'enceinte, on observe aussi une 
ciselure verticale en retrait du même type près de la tour 2 (cf. $B C H, 119,1995$, p. 770, fig. 11 ; Atlas d'Apollonia, p.178). Cette partie du rempart pourrait appartenir au même programme édilitaire. L'hypothèse de l'usage de blocs du rempart remployés dans le mur nord du portique ou dans les fondations des bases est moins vraisemblable. Le parallèle morphologique offert par le rempart est au sud-est de la colline 104 permet de formuler l'hypothèse d'une élévation de briques cuites pour le grand portique. Il reste difficile de savoir s'il s'agit d'une véritable plumée fonctionnelle, indice d'inachèvement du ravalement du monument, ou bien d'une plumée décorative, comme le sont par exemple les tenons de bardage non ravalés du portique dorique à absides au nord-est du monument des agonothètes dans le centre monumental d'Apollonia (cf. Atlas d'Apollonia, p. 199).

De nouveaux fragments architecturaux appartenant probablement au portique ont été mis au jour dans les fosses et tranchées de spoliation des secteurs 15 et 16. Mentionnons seulement, dans le secteur 16, un grand fragment de statuette féminine en calcaire (fig. 15) trouvé près de la base 4 .

Fig. 15 - Apollonia d'lllyrie. Statuette féminine en calcaire (secteur 16).

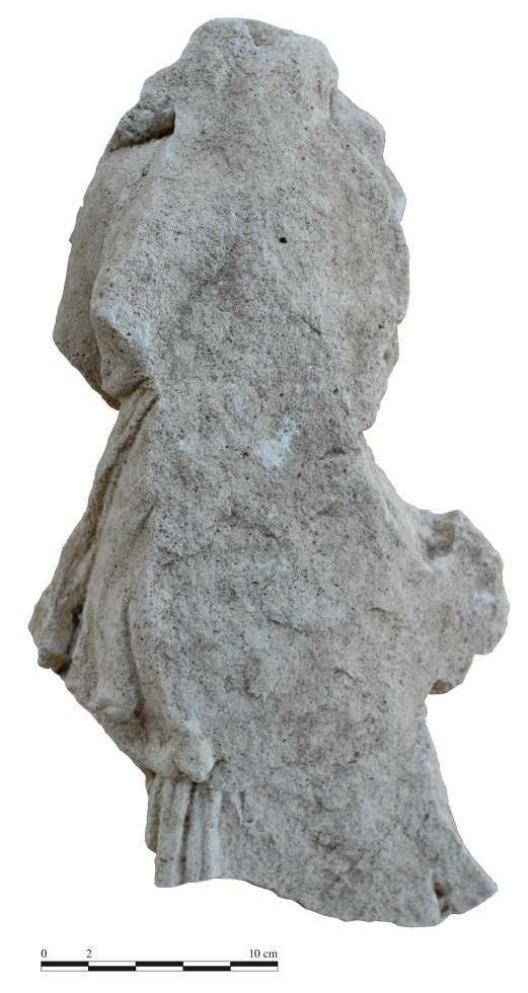

Le personnage est vêtu d'un chitôn long à apoptygma. Il peut s'agir d'Artémis, bien attestée à Apollonia, ou de la Victoire, Nikè. Elle provient peut-être d'un acrotère central du portique. 


\section{LE SECTEUR G DANS LA VILLE BASSE (J.-L Lamboley, A. Skenderaj, A. Dimo, avec la participation de $A$. Buqinca, C. Delhomme, L. Jaupaj)}

Les travaux se sont répartis entre deux équipes : celle chargée de la fouille de l'extrémité occidentale de l'égout (secteur 1, carrés 2920-2935/765-780) sous la responsabilité d'A. Skenderaj (Institut archéologique de Tirana) et celle chargée de la fouille du bâtiment au sol mosaïqué (secteur 4, des carrés 2985/765-770 jusqu'au carré 3005/755) sous la direction de A. Dimo (Parc Archéologique d'Apollonia). Il faut y ajouter l'équipe des restaurateurs des mosaïques sous la direction d'A. Islami de l'Institut des Monuments de Tirana (fig. 16).

Fig. 16 - Apollonia d'Illyrie. Planimétrie du secteur G.

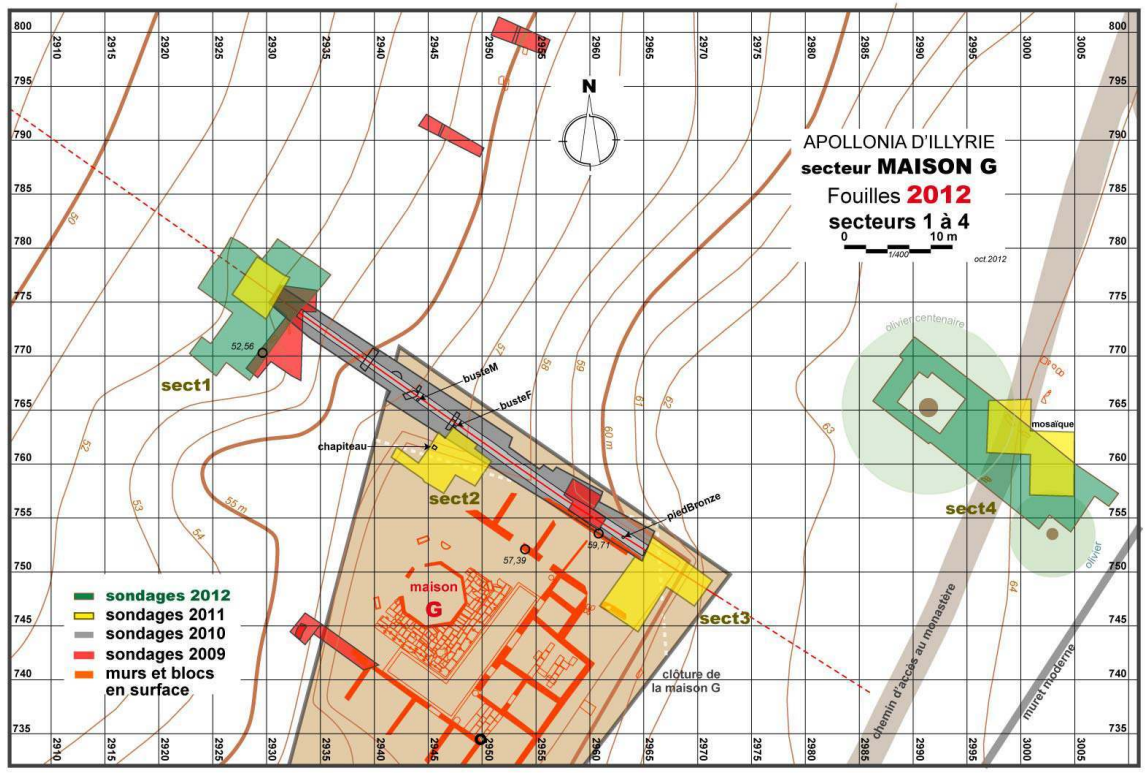

\section{L'extrémité occidentale du grand caniveau (fig. 17-18)}

La campagne de l'an dernier avait déjà identifié un carrefour entre le grand caniveau de direction est-ouest identifié dès 2009, et un caniveau transversal de direction nord-sud. Il s'agissait cette année de voir si cet égout transversal se poursuivait vers le nord, et d'élargir la zone de fouilles pour avoir une vision plus claire de l'ensemble. 
Fig. 17 - Apollonia d'Illyrie. Plan général des structures.

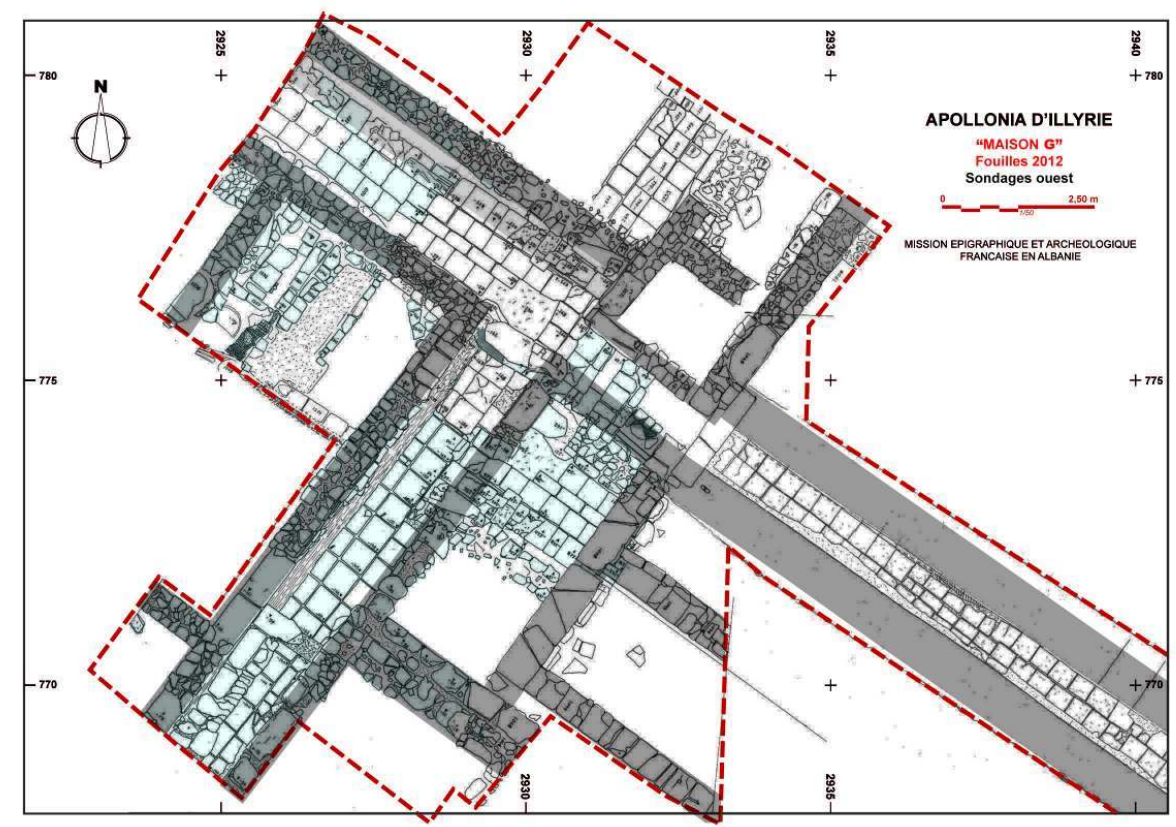

Fig. 18 - Apollonia d'Illyrie. Vue générale de l'extrémité occidentale du grand égout.

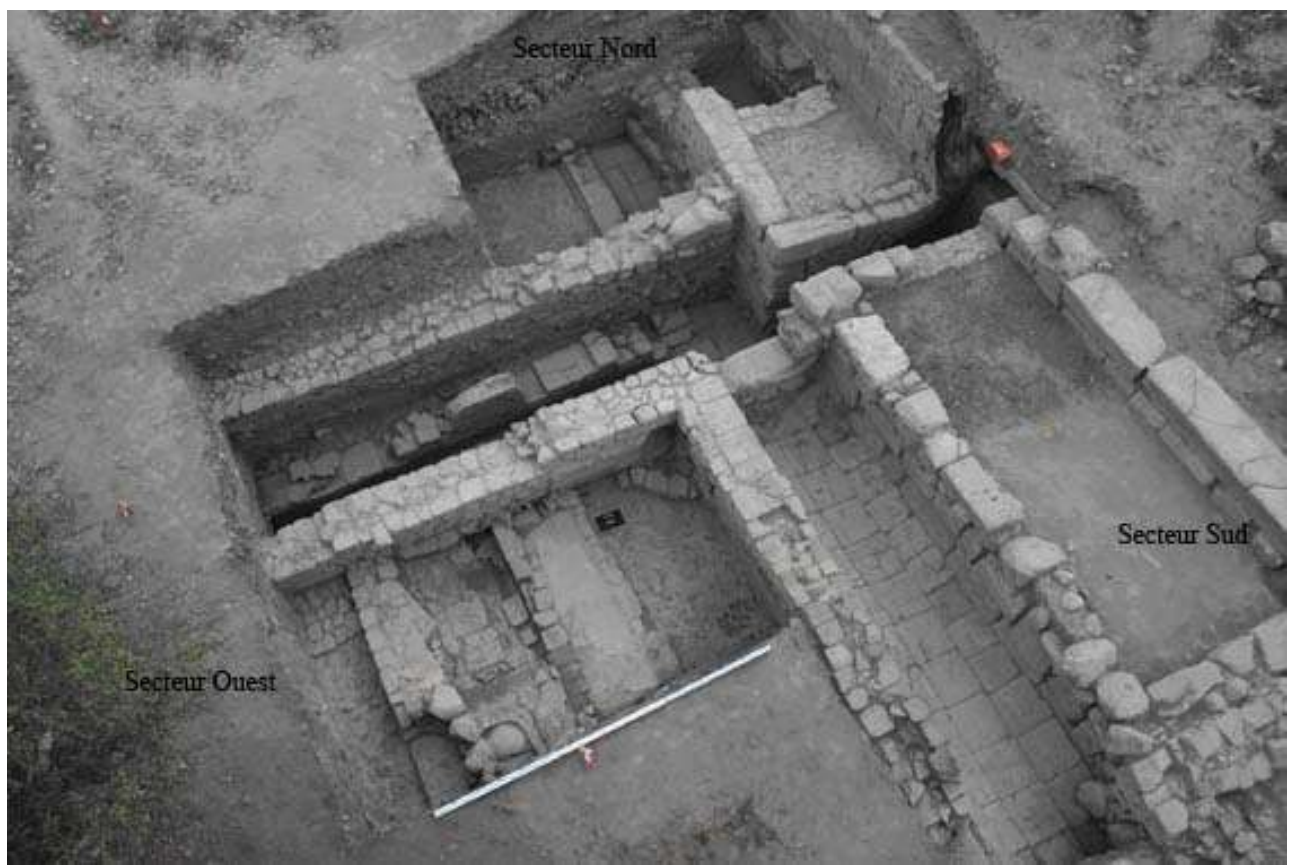

\section{Au sud}

43 Au sud, le sondage a été élargi dans les carrés 2925/765-770. Le caniveau US 1194 se poursuit bien dans cette direction en présentant toujours une épaisse couche de mortier hydraulique à l'ouest le long du mur 1107. Les dalles centrales du caniveau mesurent toutes $48 \times 48 \mathrm{~cm}$ et correspondent donc à une réfection de l'époque romaine, ce qui 
explique pourquoi ce caniveau se situe à une cote supérieure par rapport au caniveau principal dont la première phase est d'époque hellénistique ${ }^{1}$. Par contre plusieurs dalles latérales appartiennent au module hellénistique de $38 \times 38 \mathrm{~cm}$ et sont donc en remploi.

On observe toujours la même différence de structure entre le mur ouest 1107, aux fondations constituées de blocs de grès bien réguliers avec une élévation en fragments de briques liés par du mortier, et le mur est 1195 constitué de blocs de grès beaucoup plus irréguliers; dont beaucoup semblent remontés aux assises supérieures, et sans aucune trace d'élévation en brique. Cette différence s'explique par le fait que le mur ouest du caniveau correspond au mur oriental d'une nouvelle maison, alors que son mur est apparait comme un simple mur de terrassement; il n'y a en effet aucun élément d'habitation dans l'espace compris entre les murs 1008 et 1195 (fig. 18-19).

Fig. 19 - Apollonia d'Illyrie. L'égout latéral 1194.

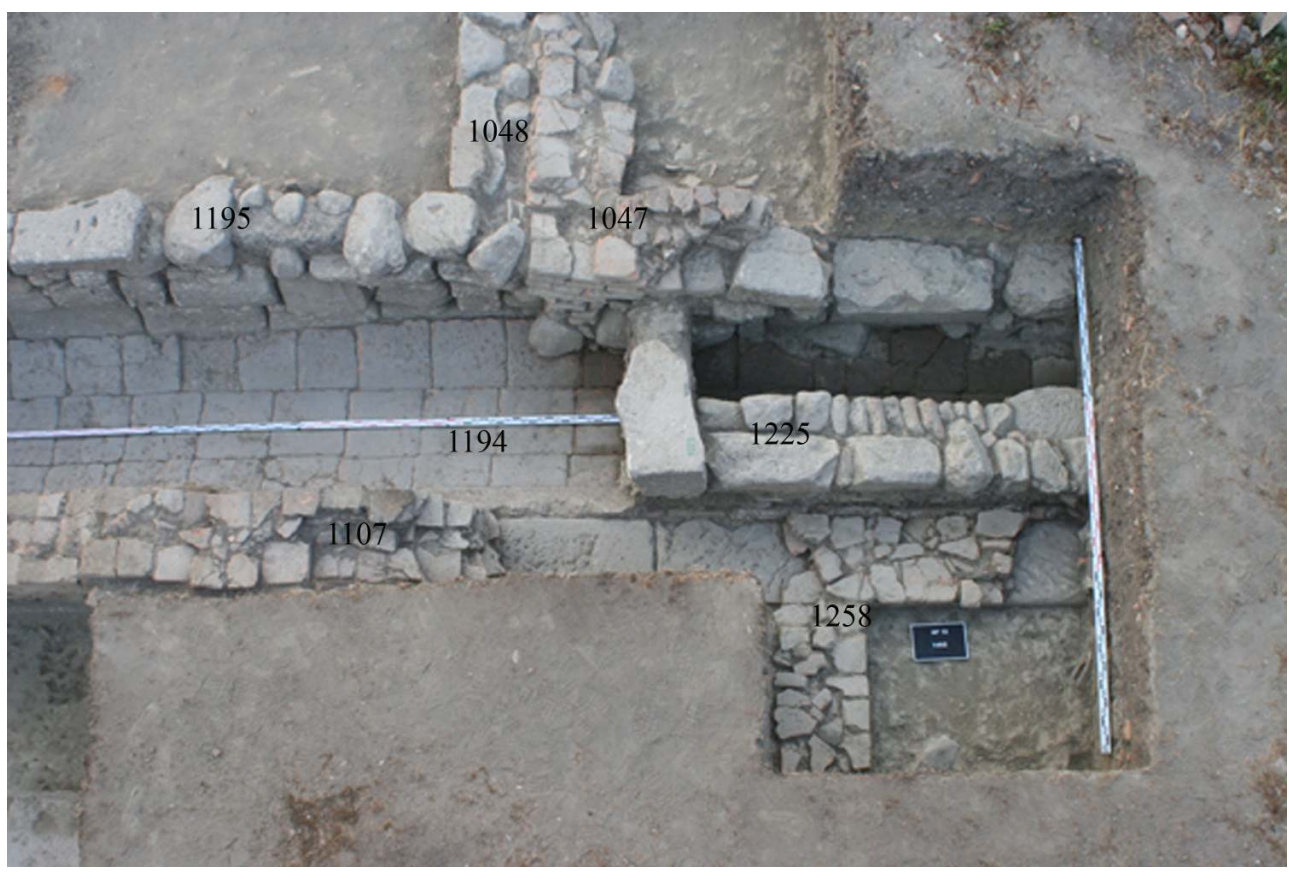

Dans le carré 2920/770, la fouille a libéré un nouveau mur en fragments de briques (US 1258) qui appartient à la maison et vient s'intégrer à l'élévation en briques du mur 1107. Ce mur permet d'identifier une première pièce à l'angle nord-est de la maison. Sur le côté opposé, à cheval sur les carrés $2925 / 765$ et 7701, ont été libérés deux murs parallèles, US 1047 et 1048, déjà identifiés lors des campagnes précédentes au moment du dégagement du mur 1008. Il a pu être établi que l'extrémité ouest du mur 1048 rentre dans le parement est du mur 1195 et lui est donc solidaire, alors que l'extrémité ouest du mur 1047 s'appuie au parement est du mur 1195 qu'il a détruit, et lui est donc postérieur. Cette situation a été confirmée par le fait que le mur 1047 fait un retour vers le sud qui recouvre le mur 1195 fortement détruit à partir de cet endroit. Le mur 1048 étant constitué seulement de fragments de blocs de grès, alors que le mur 1047 présente une élévation en fragments de briques, on avait déjà là un indice de deux périodes différentes. Le mur 1047 correspond en fait à l'angle d'un édifice postérieur qui est venu s'installer sur les murs de terrassement et qui prend également appui sur le dallage du caniveau. À ce moment, le caniveau ne peut plus être en exploitation car les eaux auraient emporté les pierres de fondation en appui sur son bord oriental. L'US 1117 recouvrant le caniveau 
et s'appuyant aux fondations du mur 1047 est donc contemporaine de la construction de ce mur. La couche supérieure, US 1110, correspond à une recharge postérieure servant sans doute à réparer l'érosion causée par le ruissellement des eaux. Sur cette recharge a été construit un nouveau mur (US 1225) parallèle au 1047, fait d'éléments en remploi, qui rétrécit la largeur du caniveau: un bloc provenant peut-être de la destruction du mur 1107 est placé en travers, le muret 1225 prenant appui sur sa face arrière et se prolongeant en appui le long du mur 1107 (fig. 18). Un espace d'une trentaine de centimètres est laissé de l'autre côté, en avant du mur 1047/1195, et l'écoulement des eaux se faisait dans cet espace directement sur la couche de terre US 1110.

C'est donc quatre phases qui ont pu être identifiées dans cette partie de la fouille; la première phase hellénistique (vraisemblablement au cours du $\mathrm{II}^{\mathrm{e}}$ siècle avant $\mathrm{J}$.-C.), puis la surélévation avec le muret de briques installant le caniveau 1194 (époque impériale), le mur 1048 qui condamne l'égout (courant III ${ }^{\mathrm{e}}$ siècle), et enfin le rétrécissement avec la dalle transversale et le muret 1225.

Toujours dans ce secteur sud de la fouille, l'angle des murs 1195 et 1045 a dû faire l'objet de travaux de restauration et de consolidation exécutés par l'Institut des Monuments de Tirana. Cette opération longue, périlleuse et couteuse..., a permis de sécuriser toute la zone, et d'accéder à l'espace très étroit où passe le collecteur principal 1017 au débouché du mur 1008, entre les murs 1045 au sud et 1200 au nord.

\section{À l'ouest}

À l'ouest, il s'est agi de dégager l'angle de la maison et la poursuite vers l'ouest du collecteur principal 1017 dans le carré 2925/775 (fig. 18, 20).

Fig. 20 - Apollonia d'Illyrie. La salle de bains à l'angle nord-est de la maison.

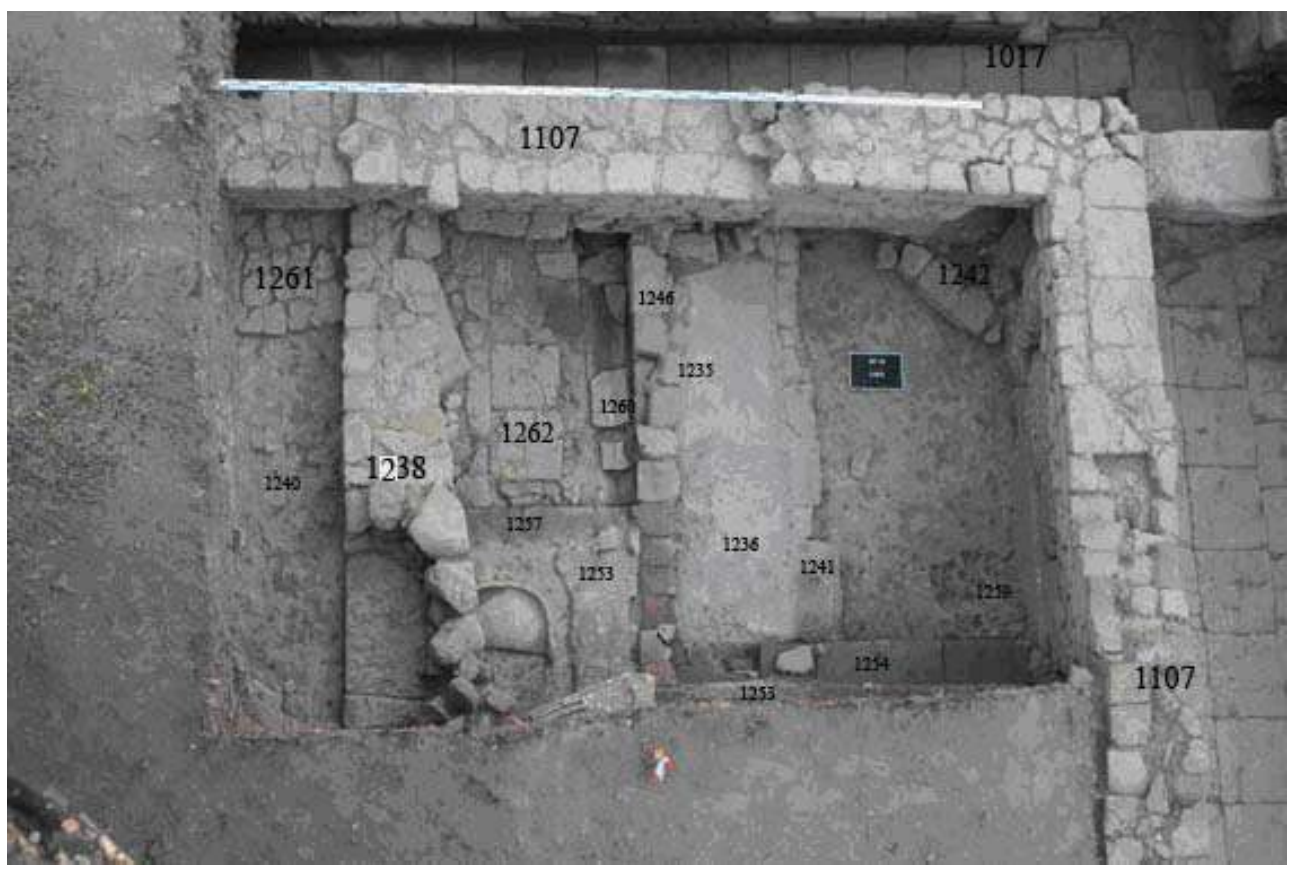

Le caniveau a été dégagé sur une longueur de 3 mètres supplémentaires par rapport à l'an dernier, mais sans apporter de données nouvelles ; on observe seulement un changement dans l'agencement des dalles de fond : la file centrale de demi-briques disparaît, et il n'y a 
plus que deux files de briques de module $48 \times 48$ sur la file nord, et 34 x 38 sur la file sud. Cela s'explique par l'avancée des blocs de fondations du mur 1107 de la maison qui rétrécissent l'espace. On observera le mélange des deux modules de briques qui montrent que l'état actuel n'est pas l'état orignal.

Le sondage a surtout été intéressant par le matériel du II siècle après J.-C. retrouvé dans le caniveau, notamment la plaque d'os travaillée en haut relief représentant Héraclès volant le trépied delphique et poursuivi par Apollon dont la finesse d'exécution est remarquable (fig. 21), plusieurs lampes à huile à décor figuré, dont l'une, à caractère érotique (fig. 22), a été l'attraction de la presse et des visiteurs pendant toute la campagne..., et un manche en os avec tête de bélier (fig. 23) pour ne citer que les pièces les plus remarquables. Il a également permis de dégager l'angle nord-est de la maison.

Fig. 21 - Apollonia d'Illyrie. Relief en os avec Héraclès volant le trépied delphique.

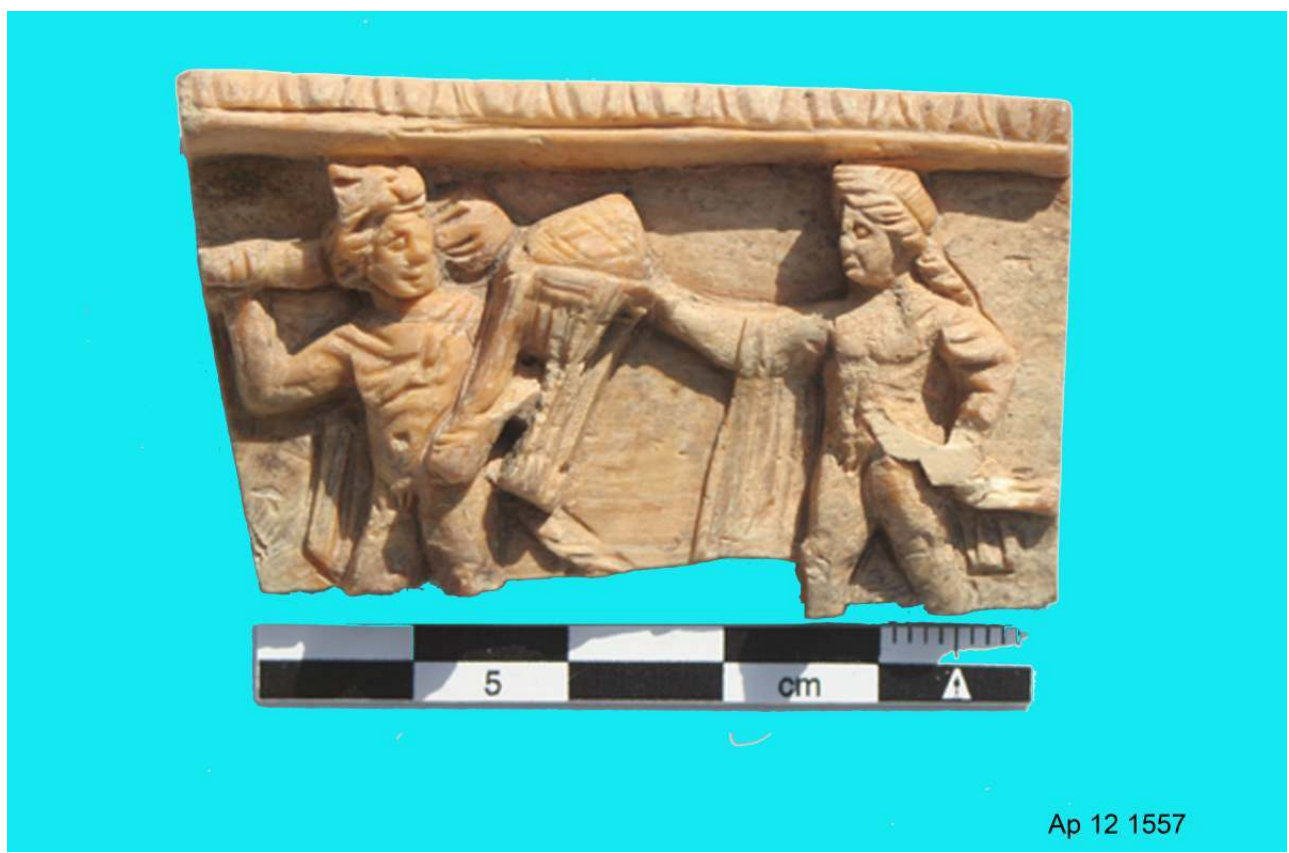


Fig. 22 - Apollonia d'Illyrie. Fragment de lampe à huile avec scène érotique.

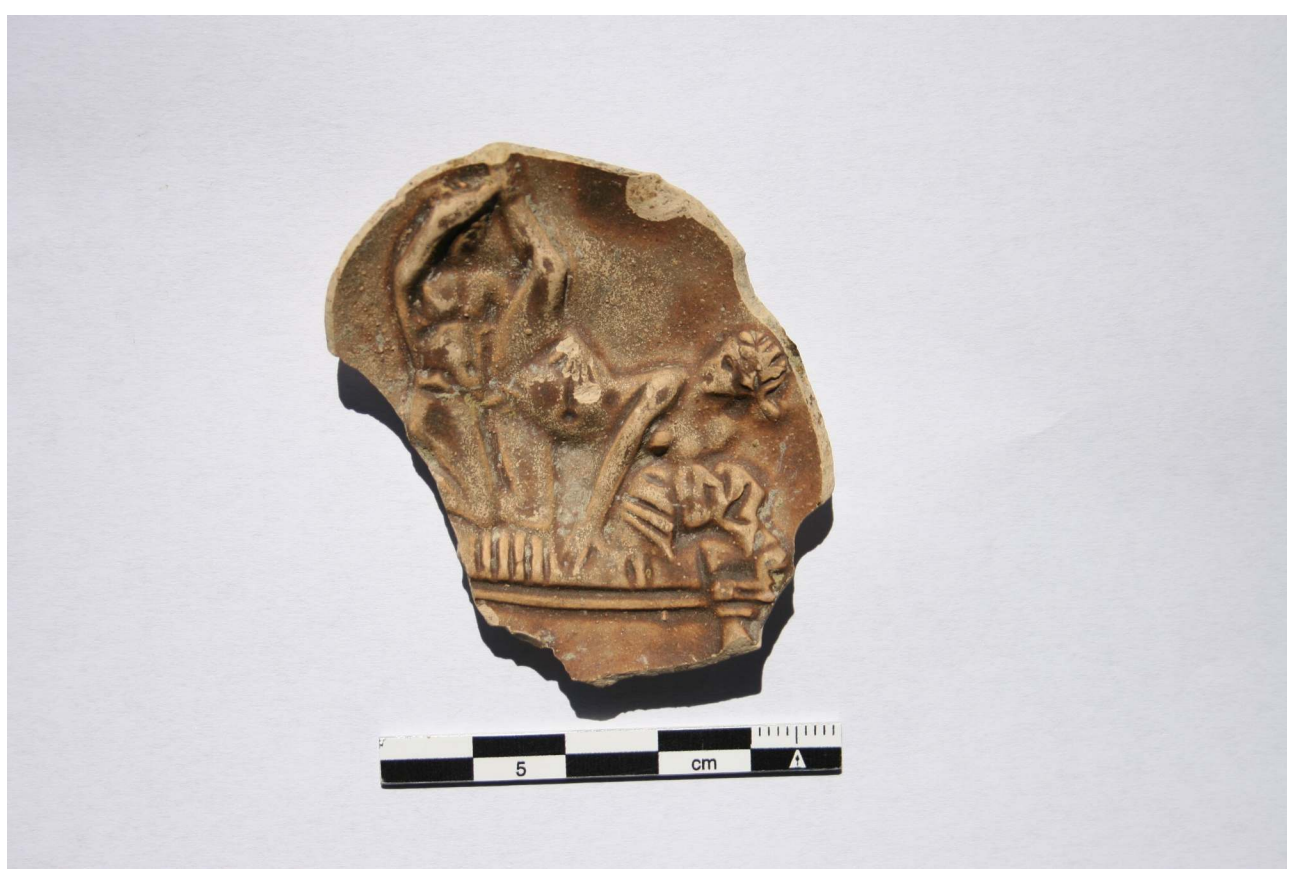

Fig. 23 - Apollonia d'Illyrie. Manche en os avec tête de bélier.

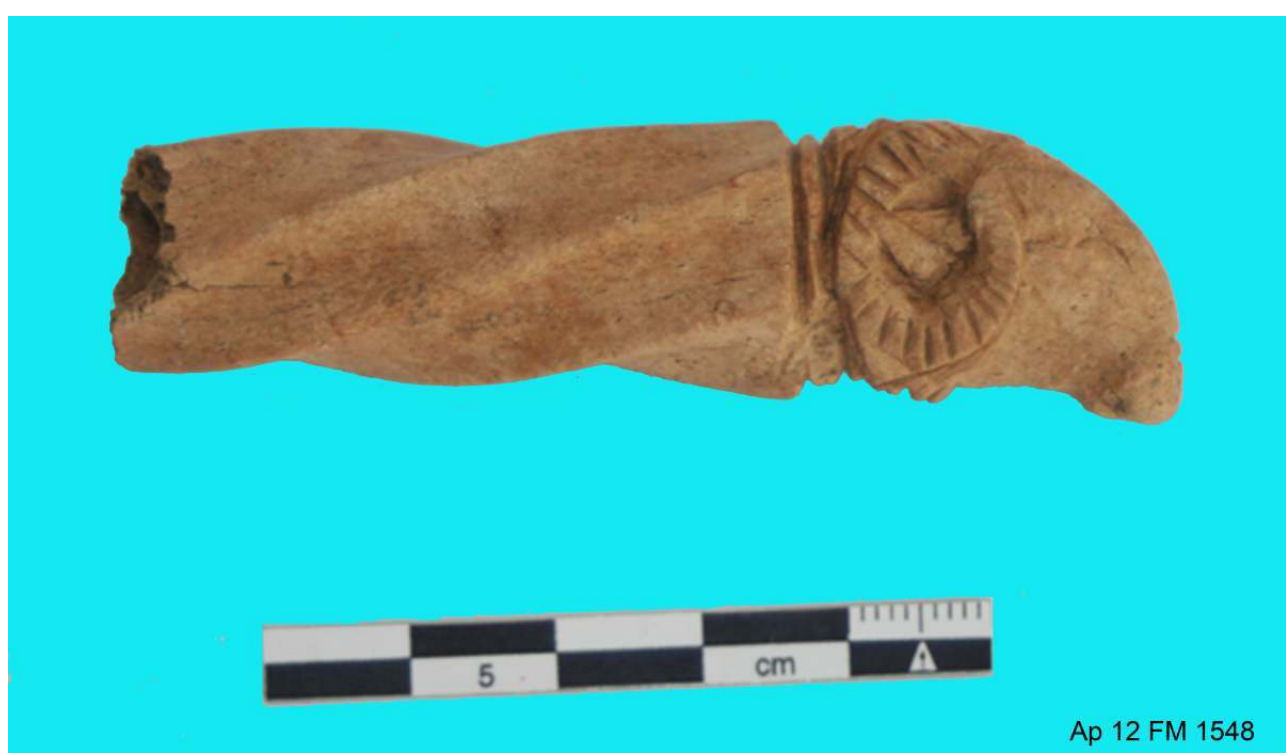

51 La surprise a été la complexité de l'agencement de la pièce qui a pu être fouillée seulement à son extrémité nord et que l'on peut identifier, à cet endroit, comme une salle de bain. Le dégagement des murs 1238 au nord et 1258 au sud permet de reconstituer une grande pièce rectangulaire de 6,30 x 3,20 m. mais avec des subdivisions internes dont certaines doivent correspondre à des réaménagements. Le muret en briques 1254 (quatre assises conservées) qui se connecte à angle droit avec le muret en briques 1246, sert de cloison à un corridor pavé en tesselles de briques de $5 \times 5 \mathrm{~cm}$ (US 1253) qui après le coude donne accès à une petit baignoire-sabot ou bain de siège dont seule la partie inférieure de la vasque est encore en place. La cloison ouest est constituée par le mur 1238. Au nord la vasque est séparée par le muret 1257 d'un espace dallé (US 1262) qui se termine le long de 
la cloison 1246 par une canalisation formée de tuiles creuses (US 1260). À noter que le pavement en tesselles 1253 présente à son extrémité nord un ressaut en cocciopesto avec une ouverture en forme de bec qui permet l'écoulement des eaux dans la canalisation 1260. Une observation importante est que cette canalisation qui va droit dans le mur 1107 ne trouve pas d'orifice traversant le mur. Il est donc clair que dans sont état actuel l'élévation en briques du mur nord 1107 est une réfection plus tardive faite à un moment où la canalisation de la pièce n'était plus en usage. On a aussi la preuve que le grand caniveau servait à recueillir non seulement les eaux de pluies, mais aussi les eaux usées venant des maisons.

À l'est du mur 1236 on a une surface en cocciopesto très compact (US 1236), endommagée à son extrémité nord le long du mur 1107 par une file de briques interrompue par un orifice permettant un écoulement, mais là encore le mur 1107 est plein. Cet espace est délimité à l'est par un nouveau muret en brique US 1241, parallèle au mur 1246. Au sud, la limite n'est pas très nette car le mur 1241 s'arrête avant le mur 1254. Les dimensions maximales sont $2,20 \times 0,60 \mathrm{~m}$. Il faut noter qu'on a les restes d'un départ d'élévation en cocciopesto sur les deux côtés longs. Il pourrait donc s'agir non pas d'un sol mais du fond d'une sorte de bassin permettant d'alimenter en eau la salle de bain. L'espace restant situé entre le mur 1241 et le mur oriental de la maison ne présente qu'un sol en terre battue avec à l'angle du mur 1254 la présence d'un foyer (US 1259) où a été retrouvé encore en place un vase à feu quasiment intact. Les murs à l'angle nord-est sont recreusés à l'intérieur pour ménager un espace de rangement bien délimité par un petit muret en diagonale (US 1242). On a donc une cohérence fonctionnelle de ces différents espaces : à l'est une zone de service avec le foyer permettant de chauffer l'eau, au centre un bassin assurant l'alimentation en eau, à l'ouest le bain de siège avec la canalisation permettant l'évacuation des eaux usagées, au sud le corridor donnant accès à l'ensemble.

Pour être complet il faut signaler que le mur 1246 a été modifié dans un second temps lorsque le bassin central n'est plus en usage ; en effet il est doublé par le mur 1236 qui empiète sur le fond en cocciopesto, et qui semble se connecter avec la structure en brique signalée au nord du bassin. Enfin, à l'angle externe des murs 1107 et 1238, ont pu être libérés les restes d'un de pavement en fragments de briques US 1261.

Toutes ces structures ont été retrouvées sous une épaisse couche de destruction contenant du matériel du $\mathrm{II}^{\mathrm{e}}-\mathrm{III}^{\mathrm{e}}$ siècles après $\mathrm{J}$.-C et qui correspond au moment de l'abandon de l'habitat et du comblement des caniveaux. L'hypothèse d'une destruction à la suite d'un tremblement de terre trouve donc encore un élément de confirmation. Toute la zone subit ensuite des perturbations, sans doute à l'époque médiévale, comme le prouvent la présence de deux fosses dont l'une a crevé le mur 1238.

\section{Au nord}

Au nord (carré 2930/775) l'objectif était de retrouver la suite du caniveau 1194 de l'autre côté du collecteur principal. Le travail de fouille a été très long compte tenu de l'épaisseur à cet endroit des couches de destructions et des remblais. Finalement le caniveau a bien été retrouvé, et l'intérêt de ce sondage a été aussi de révéler une situation de murs de terrassement qui n'est pas symétrique de la situation connue au sud (fig. 18, 24). 
Fig. 24 - Apollonia d'Illyrie. Le secteur nord de légout 1233 (vu de l'ouest).

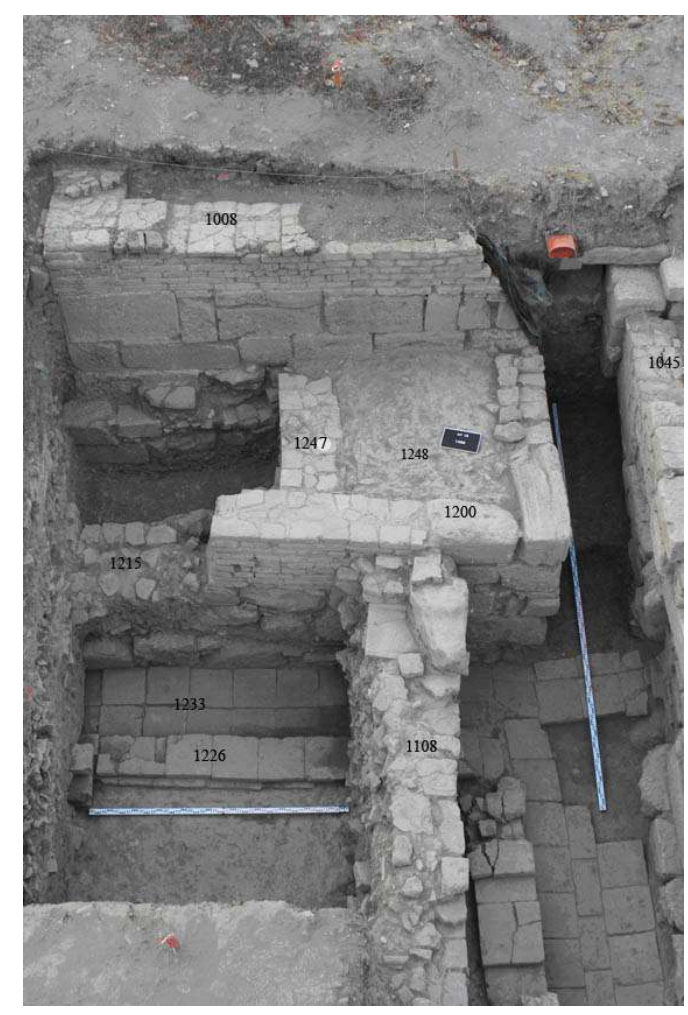

La suite du mur 1008, le plus à l'est (déjà dégagé en partie en 2009 où on avait relevé son éventrement par une fosse médiévale encore visible sur la fig. 24), a été libéré jusqu'à un retour vers l'est, juste à la limite nord du sondage. Ses fondations semblent reposer sur les restes d'une muraille précédente qui elle se poursuit vers le nord dans la berme, mais présente un profil en demi cercle à l'extrémité sud. On aurait donc ici deux phases différentes. Ce retour vers l'est, à $4,10 \mathrm{~m}$ de la paroi nord du collecteur principal, accentue la dissymétrie avec le mur 1008 au sud du collecteur principal qui se poursuit vers le sud sans interruption, créant ainsi une grande terrasse avec le mur 1195. Il sera important l'an prochain d'élargir le sondage vers le nord afin de voir si le mur 1008 repart plus loin, ce qui, dans ce cas, délimiterait une sorte d'entrée.

En avant du mur 1008, a été appuyée une structure angulaire en forme de bastion qui renforce l'extrémité du débouché du collecteur principal; elle est constituée du mur principal 1200 dont on n'a pas distingué les deux ailes sud et ouest car l'angle montre une parfaite connexion des deux courtines. Ce mur est donc le symétrique des murs 1045 et 1195 au sud, mais son angle n'a pas subi de destruction comme c'est le cas au sud. Le mur ouest s'interrompt également vers le nord, comme le mur 1008, mais à 2,76 m seulement de l'angle du caniveau; le parement nord de l'extrémité est très soigné, et il ne s'agit donc pas d'une destruction. À $45 \mathrm{~cm}$ avant son extrémité nord, le mur ouest est renforcé à l'arrière par un mur transversal, de direction est/ouest (US 1247), qui n'arrive pas jusqu'au parement ouest du mur 1008 car ses fondations ont buté contre celles en saillie de la première phase du mur 1008. Les fondations en blocs de grès du mur 1200 reposent elles aussi sur les restes d'une fondation précédente qui sert de paroi est au caniveau (US 1233) dont le dallage a été retrouvé à la cote moyenne -260. 
Ce caniveau, dans l'état actuel est moins large que le caniveau sud 1194 ou que le collecteur principal 1017 ; il est constitué simplement de deux files de briques de module hellénistique et ne dépasse pas les $77 \mathrm{~cm}$ de large (contre $97 \mathrm{~cm}$ pour les caniveaux 1194 et 1017). Son profil est presque plat, à la différence des autres qui ont un profil en $U$ ou en $\mathrm{V}$ beaucoup plus affirmé. La connexion avec le collecteur central n'a pas pu être faite à cause de la présence du mur tardif 1108 que l'on a renoncé à démonter du fait de son très mauvais état de conservation. Le mur est du caniveau 1233, on l'a vu, est constitué par les blocs de fondations d'une première phase du mur 1200; par contre son mur ouest (US 1226) est constitué par un muret de briques de module d'époque romaine qui vient se connecter, sous le mur 1108, au muret nord (US 1160) du collecteur principal fait lui aussi de briques de $54 \times 54 \mathrm{~cm}$. Il est donc vraisemblable que ce mur 1226 est une réfection d'époque romaine qui vient se poser sur le bord occidental du caniveau qui dans sa première phase hellénistique était donc plus large. Cela explique pourquoi le mur 1160 présente comme un retour interrompu à quelque $40 \mathrm{~cm}$ de son angle actuel. Ce retour coïncidait avec l'alignement du mur ouest du caniveau 1233 situé $40 \mathrm{~cm}$ plus à l'ouest dans sa première phase. Il est probable que la réfection qui a conduit à l'état actuel s'est faite au même moment que la mise en place du bastion d'angle 1200 qui vient remplacer une structure antérieure dont on n'a plus que les fondations.

Il faut noter aussi que l'espace libre au nord de l'extrémité du mur ouest 1200 a été obturé dans un second temps par un muret composé de matériaux hétéroclites très endommagés et ne reposant sur aucune fondation solide (US 1215). Là aussi l'élargissement du sondage vers le nord devra permettre de trouver l'extrémité de ce muret et le nouveau départ du mur 1200. On peut se demander si tous ces espaces libres combles et obturés après des périodes de destruction, n'étaient pas occupés par des escaliers en bois qui permettaient de communiquer entre les caniveaux et les terrasses supérieures.

\section{Le bâtiment à mosaïque (fig. 25)}


Fig. 25 - Apollonia d'Illyrie. Plan de l'édifice à mosaïque.

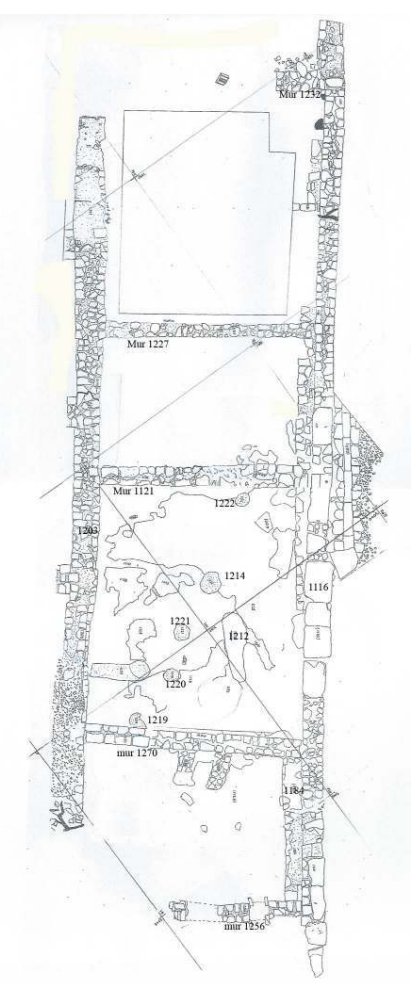

60 L'extension de la fouille vers le sud dans les carrés 2995-3000/765 a permis de retrouver le bord méridional de l'espace mosaïqué dont on connaissait depuis l'an dernier le bord septentrional bordé par une rue. La largeur de la pièce peut ainsi être établie à 5,10 m entre les murs 1116 au nord et 1203 au sud (fig. 26). 
Fig. 26 - Apollonia d'Illyrie. Le sol en mosaïque (après restauration).

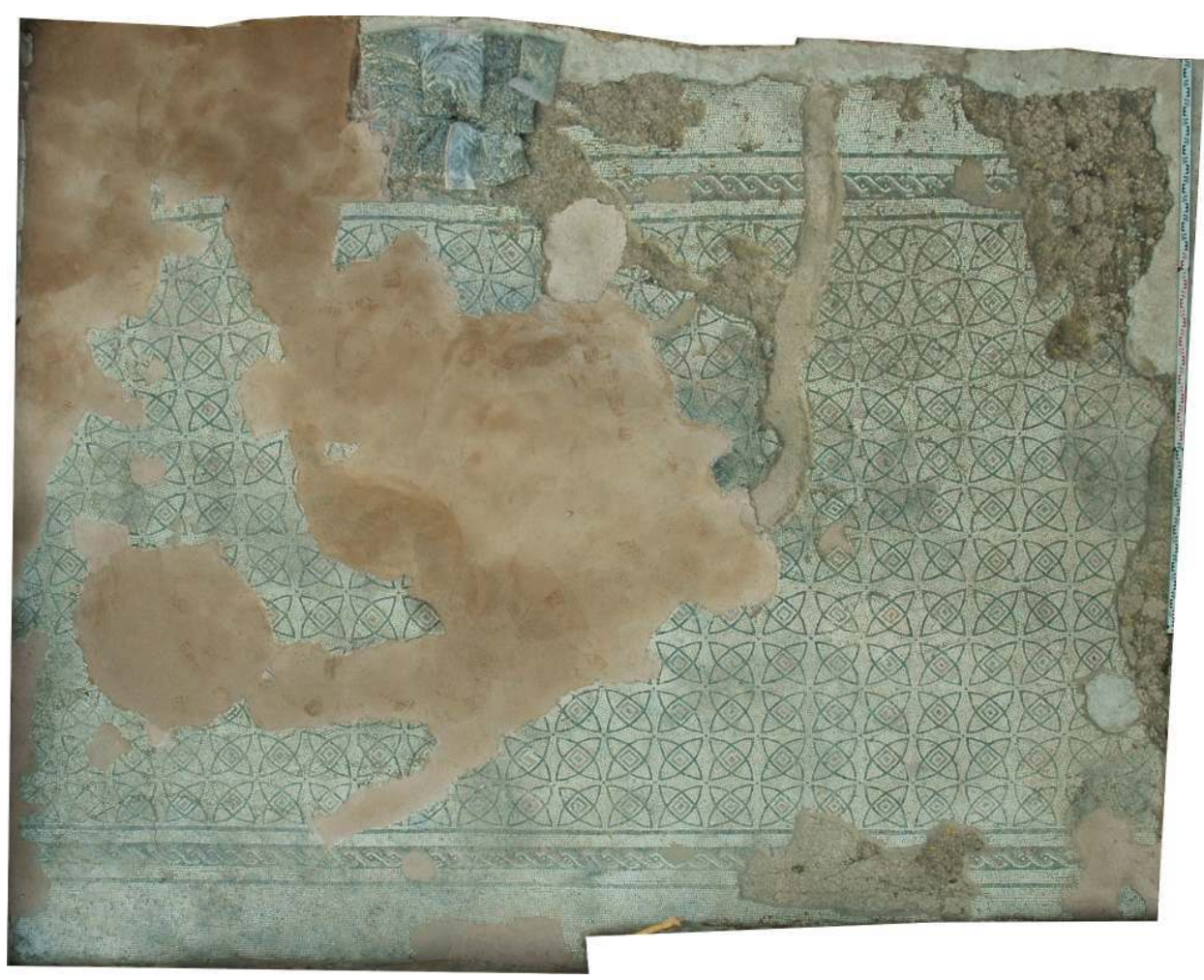

Le temps a manqué pour libérer la mosaïque sur toute sa surface à l'extrémité orientale du sondage où elle est encore conservée, car à cet endroit elle est recouverte par une épaisse couche de chaux très compacte; par ailleurs la mosaïque - très fragilisée par le passage des voitures - a été consolidée au fur et à mesure de sa découverte, et les travaux de restauration étant forcément plus lents, il ne convenait pas de dégager des surfaces qui n'auraient pu être consolidées avant la fin du chantier. Dans la partie dégagée cette année, à l'angle sud-ouest du carré 3000/760 a été découverte une tombe à fosse (US 1212) contenant les restes d'un soldat turc de la première guerre mondiale identifiable grâce à son briquet de fusil (fig. 27). Juste à côté on retrouve le trou d'obus déjà identifié l'an dernier (US 1193). 
Fig. 27 - Apollonia d'Illyrie. La tombe et les fosses dans le sol de l'édifice à mosaïque.

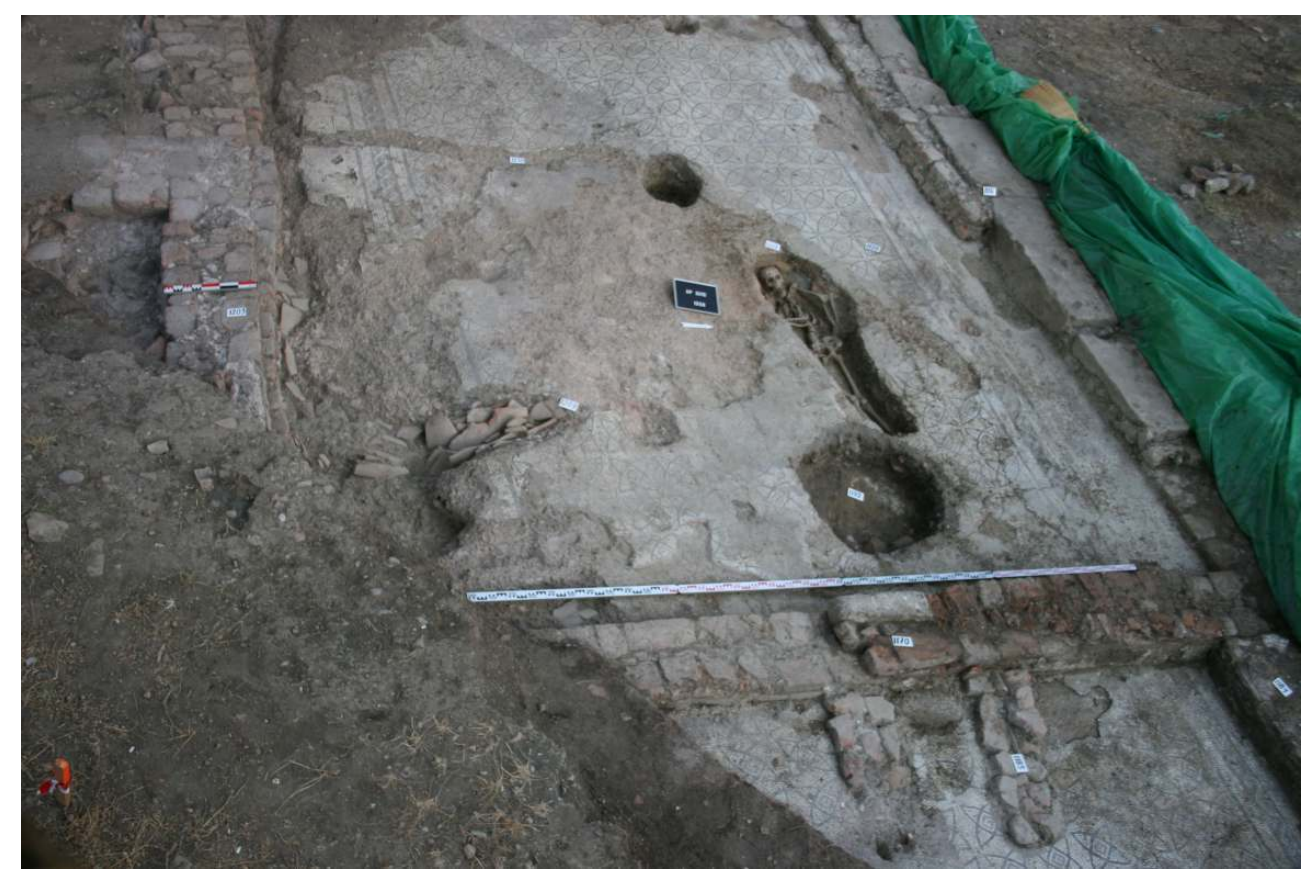

Il a été également possible d'identifier une série de cinq orifices qui ont cassé la mosaïque, et qui sont grossièrement alignés sur un axe est-ouest : US 1219, 1220, 1221, 1214 et 1222 (visibles sur le plan fig. 25 et sur la photo fig. 27). Il peut s'agir de trous de poteaux d'une construction d'époque médiévale.

Les quatre murs transversaux qui ont été dégagés (US 1256, 1170, 1121, et 1227 d'est en ouest) sont des murs qui appartiennent à une deuxième phase de l'édifice au cours de laquelle la mosaïque initiale est toujours en usage, mais l'espace est alors découpé en plusieurs pièces contiguës, alors que dans la première phase la mosaïque constitue un sol continu de plus de $20 \mathrm{~m}$ de longueur. Le but de la campagne était bien sûr d'identifier l'entrée de ce bâtiment et ses limites orientales et occidentales. Aucun seuil n'a pu être identifié sur les murs nord et sud. À noter qu'au sud du mur méridional 1203 deux départs de murs perpendiculaires ont été dégagés, distant de $3,40 \mathrm{~m}$; la mosaïque est absente à cet endroit.

La fouille a ensuite été étendue vers l'ouest jusque dans les carrés 2985/765-770 de l'autre côté du grand chêne qui borde à l'ouest la route moderne. A l'extrémité occidentale du sondage, juste à l'angle nord-ouest du carré 2990/765, un retour de mur vers le sud (US 1232) a été dégagé, et celui-ci semble solidaire du mur 1116 car il a la même largeur, le même type de mortier, et des briques des assises inférieures sont communes aux deux murs (fig. 28). 
Fig. 28 - Apollonia d'Illyrie. Le retour d'angle du mur 1116 à l'extrémité occidentale de l'édifice à mosaïque.

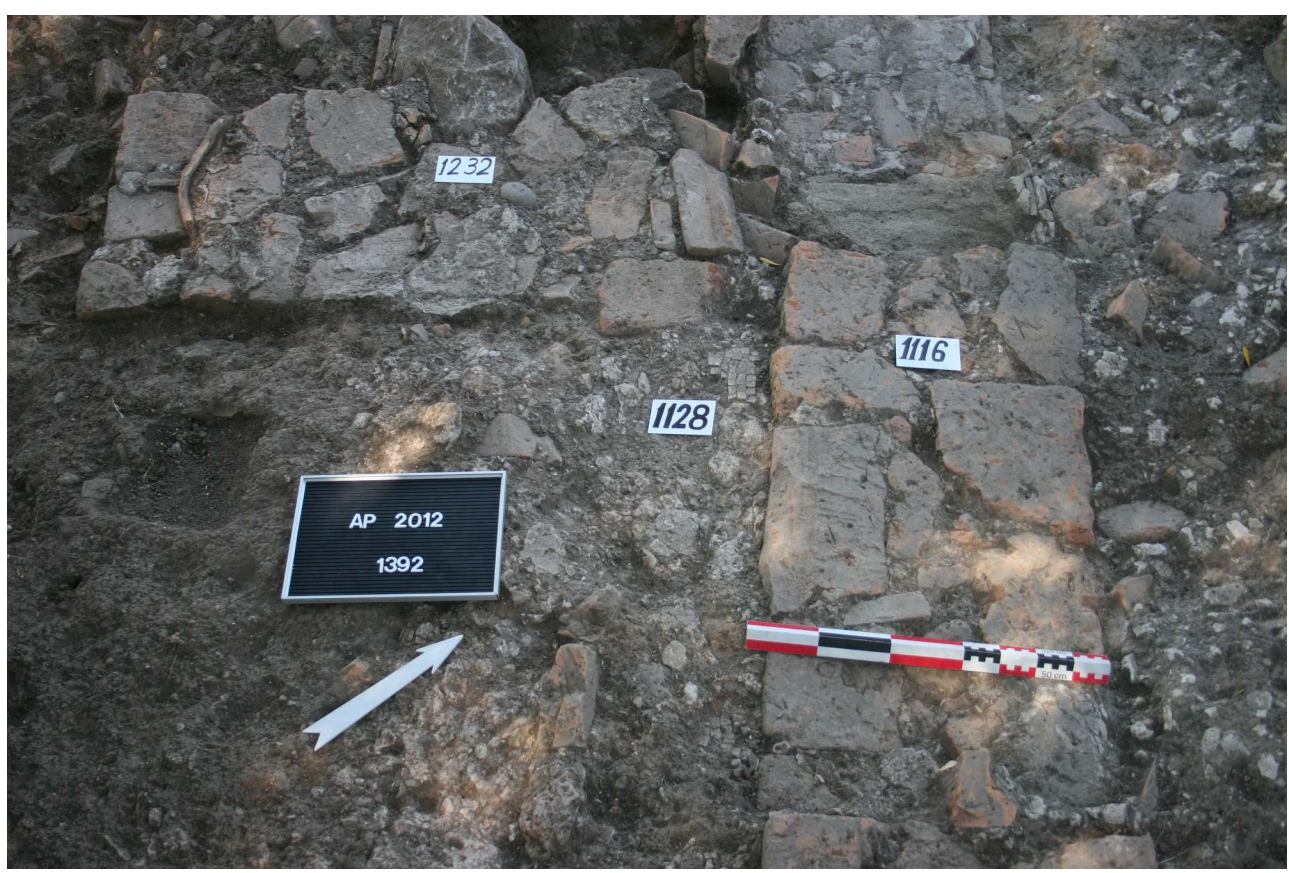

Juste après ce retour, le mur 1116 semble s'interrompre au bout de $70 \mathrm{~cm}$; cette dernière portion pourrait donc être un mur d'ante. Par ailleurs le mur 1252 s'interrompt de façon nette avec un parement sur l'extrémité sud, ce qui indique une entrée possible. Qui plus est, à l'angle interne des murs 1116 et 1252 un lambeau de mosaïque est miraculeusement conservé (bien visible sur la fig. 28), alors qu'à l'angle externe, il n'y a aucune trace même d'un radier. Malheureusement toute la partie sud est complètement détruite du fait de la présence du grand chêne, et on ne peut donc établir l'aile symétrique. La présence d'un fragment de pilastre ionique juste à l'emplacement de cette éventuelle entrée, est un indice supplémentaire. Il faut enfin remarquer que l'on arrive à cet endroit à la hauteur de la limite orientale de la maison romaine à atrium. Il sera donc impératif l'an prochain de continuer la fouille à cet endroit pour vérifier la présence d'une rue nord-sud. Si c'était le cas, on aurait la preuve définitive que l'entrée de l'édifice se situe bien à cet endroit.

66 Enfin, la fouille a été étendue vers l'est jusqu'au carré 3005/755. Le dégagement de l'extrémité nord du mur transversal 1256, de deuxième phase, a permis d'identifier, par la présence de cranes, quatre autres sépultures; il s'agit à coup sûr d'autres soldats trucs comme celui de la tombe 1212. Il a été décidé d'interrompre la fouille, car le dégagement de ces dépositions pouvait poser des problèmes d'ordre diplomatique avec l'État turc en droit de demander l'identification et le rapatriement des corps, problème que la Mission n'avait pas le temps de régler, et que la direction du parc ne tenait pas à affronter. La limite occidentale d l'édifice sera donc difficile à établir, d'autant qu'il ne semble pas raisonnable de prolonger la fouille dont le but était simplement de mettre à jour une structure permettant de condamner l'utilisation de la route moderne.

67 L'identification de l'édifice reste donc problématique. Dans une première phase il se présente comme un long couloir de 5,10 $\mathrm{m}$ de large, dégagé sur plus de $20 \mathrm{~m}$. Le matériel récupéré, encore en cours d'étude, ne permet pas de remonter au-delà du $\mathrm{II}^{\mathrm{e}}$ siècle 
après J.-C. Les monnaies les plus fréquentes sont celles du III siècle (monnaies en bronze de Probus). Ce bâtiment est parfaitement intégré dans le schéma régulateur hippodaméen de la ville basse, dit réseau rouge, puisque son aile nord suit une rue d'orientation est/ ouest. On a vu qu'une entrée principale pourrait être identifiée à l'ouest, à l'endroit où devrait passer une rue d'orientation nord/sud. On pourrait imaginer un édifice en pi, portique ou leschè dont on n'aurait pour l'instant que l'aile septentrionale ouvrant sur l'ouest. Cet édifice est encore en usage dans le dernier quart du IV siècle après J.-C. comme le prouve une monnaie de l'empereur Gratien retrouvée au contact de la mosaïque ; mais à cette époque, il a déjà été transformé avec les murs transversaux qui délimitent toute une série de pièces. La troisième phase d'époque médiévale est postérieure à l'abandon et à la destruction de l'édifice, et est attestée par l'épaisse couche de chaux qui recouvre toute la partie orientale et la récupération d'objets métalliques de la période du monastère. La dernière phase d'occupation de ce secteur, correspond à la nécropole au pied du monastère qui sert à enterrer des soldats morts lors de la première guerre mondiale.

\section{CONCLUSIONS (J.-L. Lamboley, F. Drini)}

Les opérations menées sur le terrain depuis 2009 ont apporté des résultats considérables pour notre connaissance de l'urbanisme de la ville d'Apollonia. Il faut d'abord rappeler la localisation de l'agora entre les deux collines, qui est une donnée tout à fait fondamentale, et l'identification dans la ville haute du réseau bleu que l'on peut qualifier de réseau régulier polycentrique (trois quartiers identifiés), avec des îlots de $20 \times 40$ pieds. Les fouilles du portique nord ont par ailleurs permis de fixer l'évolution de l'urbanisme à la limite de l'agora et d'un quartier d'habitation depuis l'époque de la fondation jusqu'à la destruction du portique aux $\mathrm{II}^{\mathrm{e}}-\mathrm{III}^{\mathrm{e}}$ siècles après J.-C. On rappellera aussi que le portique nord de l'agora avec ses 23 colonnes est le plus grand portique connu sur le territoire de l'actuelle Albanie ( 130 x 21,7 m). Il est dans la même orientation que tous les édifices du II siècle avant J.-C. identifiés lors des fouilles de 1994-2002. Dans le secteur $G$, l'identification du carrefour des caniveaux situé au cœur d'une insula permet aussi de fixer définitivement le réseau rouge de plan hippodaméen (système régulier unitaire). Les îlots mesurent ici 100 pieds. On a ainsi tout l'organisation urbaine de la ville qui est maintenant connue, avec des grandes étapes chronologiques : ainsi la deuxième moitié du II siècle avant J.-C. est un moment de grand aménagement urbanistique dans toute la ville, et la destruction du portique à la fin du $\mathrm{II}^{\mathrm{e}}$ ou début du $\mathrm{III}^{\mathrm{e}}$ siècle après J.-C. est à mettre en relation avec le comblement des caniveaux dans la ville basse et correspond sans doute à une destruction due à un séisme important. 


\section{NOTES}

1. À son embouchure le caniveau 1194, est à la cote -172 alors que le caniveau principal 11 est à la cote -264. Les cotes sont données par rapport à la cote base située à 52,56 m au-dessus du niveau de la mer.

\section{INDEX}

Mots-clés : cité grecque, cité romaine, urbanisme, évolution urbanistique, ville haute, ville basse, Illyrie, agora, portique, égout

institutions Institut archéologique d'Albanie (Centre d'études albanologiques - Tirana), Mission épigraphique et archéologique française en Albanie (Ministère des Affaires étrangères et européennes), UMR 5189 (Lyon 2-CNRS), AOROC (UMR 8546 CNRS-ENS), IRAA (USR 3155 du CNRS), École française d'Athènes, École française de Rome

\section{AUTEURS}

\section{JEAN-LUC LAMBOLEY}

Directeur de la mission épigraphique et archéologique française en Albanie, Université Lyon 2, UMR 5189 HiSoMA - jean-luc.lamboley[at]mom.fr

\section{FAÏK DRINI}

Directeur de la Mission albanaise d'Apollonia, Institut archéologique d'Albanie

\section{FRANÇOIS QUANTIN}

Université de Pau, IRAA (USR 3155 du CNRS) - francois.quantin[at]univ-pau.fr

\section{STÉPHANE VERGER}

INHA - EPHE, AOROC (UMR 8546 CNRS-ENS) - stephane.verger[at]ens.fr

\section{ALTIN SKENDERAJ}

Institut archéologique d'Albanie - askenderaj[at]yahoo.fr

\section{SAÏMIR SHPUZA}

Institut archéologique d'Albanie - saimirshpuza[at]hotmail.com 